\title{
A priori subgrid analysis of temporal mixing layers with evaporating droplets
}

Nora Okong'o, and Josette Bellan

Citation: Physics of Fluids 12, 1573 (2000); doi: 10.1063/1.870405

View online: http://dx.doi.org/10.1063/1.870405

View Table of Contents: http://aip.scitation.org/toc/phf/12/6

Published by the American Institute of Physics

\section{Articles you may be interested in}

Direct numerical simulation and subgrid analysis of a transitional droplet laden mixing layer

Physics of Fluids 12, 650 (2000); 10.1063/1.870271

A dynamic subgrid-scale eddy viscosity model

Physics of Fluids A: Fluid Dynamics 3, 1760 (1998); 10.1063/1.857955

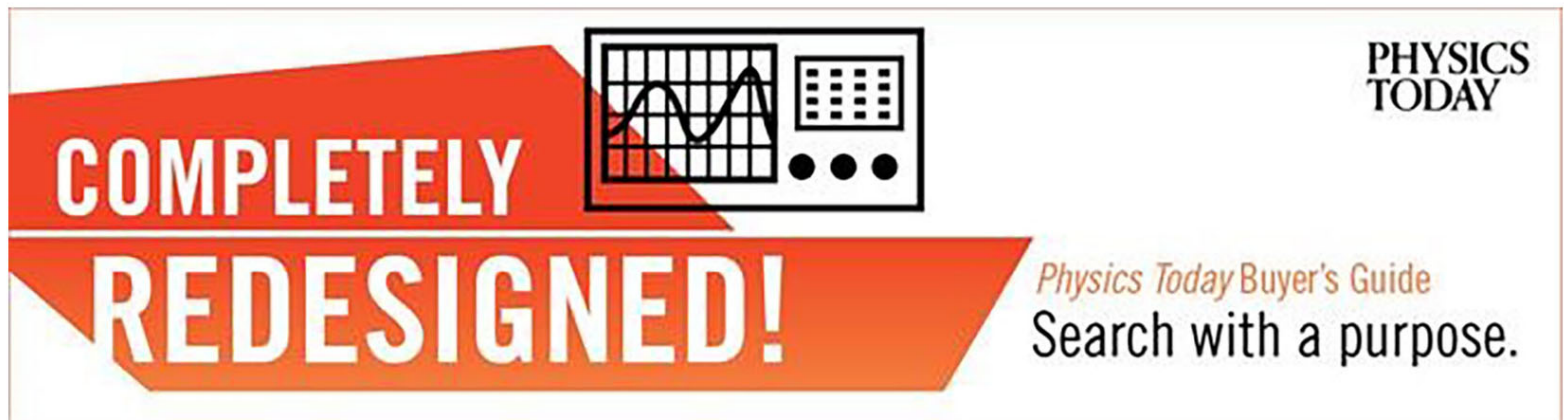




\title{
A priori subgrid analysis of temporal mixing layers with evaporating droplets
}

\author{
Nora Okong'o and Josette Bellan ${ }^{\text {a) }}$ \\ Jet Propulsion Laboratory, California Institute of Technology, 4800 Oak Grove Drive, MS 125-109, \\ Pasadena, California 91109-8099
}

(Received 23 June 1999; accepted 3 March 2000)

\begin{abstract}
Subgrid analysis of a transitional temporal mixing layer with evaporating droplets has been performed using three sets of results from a direct numerical simulation (DNS) database, with Reynolds numbers (based on initial vorticity thickness) as large as 600 and with droplet mass loadings as large as 0.5. In the DNS, the gas phase is computed using an Eulerian formulation, with Lagrangian droplet tracking. The large eddy simulation (LES) equations corresponding to the DNS are first derived, and key assumptions in deriving them are first confirmed by using the DNS database. Since LES of this flow requires the computation of droplet source terms, it is essential to obtain the unfiltered gas-phase variables at droplet locations from filtered gas-phase variables at the grid points. This paper proposes to model these unfiltered gas-phase variables at the drop locations by assuming the gas-phase variables to be the sum of the filtered variables and a correction based on the filtered standard deviation; this correction is then computed from the subgrid scale (SGS) standard deviation. This model predicts the unfiltered variables at droplet locations considerably better than simply interpolating the filtered variables. Three methods are investigated for modeling the SGS standard deviation: the Smagorinsky approach, the gradient model and the scale-similarity formulation. When the proportionality constant inherent in the SGS models is properly calculated, the gradient and scale-similarity methods give results in excellent agreement with the DNS.
\end{abstract}

(C) 2000 American Institute of Physics. [S1070-6631(00)02306-0]

\section{INTRODUCTION}

Droplet-laden turbulent flows are an inherent part of atmospheric flows such as rain and superficial oceanographic shear layers, and of industrial processes such as spray painting, ink jet printing, household and medical spray dispensing, and spray atomization and combustion in engines and furnaces. The interaction of particles and turbulence gives these flows their particular characteristics which are different from single phase flows. For example, it is well known that addition of particles to a mixing layer increases or decreases its stability depending upon the ratio of the particle diameter divided by the turbulence length scale (see Ref. 1). Moreover, when gas evolves from the particles (e.g. phase change for drops, chemical reactions for solid particles) there is an additional thermodynamic coupling related to the dynamic one (because of the added mass to the gas phase) which might change this stability boundary.

Because the particle-turbulence interaction is an integral feature of such flows, it has been the topic of much research. ${ }^{1-4}$ Large eddy simulation (LES), in which the flow field is spatially filtered, is emerging as a powerful tool in modeling unsteady turbulent flows. It is expected to be more generally applicable than Reynolds-averaged Navier Stokes (RANS), since the large scale structures are computed, and the more universal small scale structures are modeled. LES is also less computationally intensive than direct numerical

${ }^{a)}$ Electronic mail: josette.bellan@jpl.nasa.gov simulation (DNS) in which all length scales are resolved, and has the additional advantage of being able to accommodate considerably larger Reynolds numbers. Whereas much research has been devoted to LES modeling for single phase incompressible flows, only moderate attention has been given to compressible shear flows, ${ }^{5,6}$ with focus now turning two-phase flows. ${ }^{7-9}$ Several LES studies exist of particle laden homogeneous shear flows, ${ }^{8,10}$ of mixing layers, ${ }^{11}$ of particle laden channel flows, ${ }^{12-14}$ of sprays, ${ }^{15}$ and of general particle laden flows. ${ }^{7}$ However, none of those studies benefitted from an a priori SGS model; instead, the SGS model was assumed and sometimes further validated with data. In the present study, we develop an a priori SGS model for drop laden mixing layers with phase change.

The success of the LES approach crucially depends upon the fidelity of the SGS in modeling the small scales by using only the values of the variables at the larger scale, which are the only quantities available in the LES context. It is noteworthy that due to the necessity of (accurately) computing the source terms resulting from the drop-gas interaction, SGS modeling for two-phase flows requires the modeling of unfiltered gas variables at the drop locations. In simplistic models, the filtered variables are substituted for the unfiltered variables, but this expeditious choice is made only when DNS databases or empirical data are not available to guide SGS modeling; this assumption may be substantially inaccurate for droplets. With an increasing body of DNS computations, ${ }^{2,3,16-22}$ it is now possible to assess SGS quan- 
tities at moderate Reynolds numbers, with good prospects for devising SGS models.

In a recent DNS, Miller and Bellan ${ }^{19,20}$ generated a database for droplet laden three-dimensional mixing layers with phase change. The authors used the terminology 'DNS' to refer to computations in which all length scales of the gas-phase are resolved but the effect of the gas on each droplet (drop sizes are smaller than the Kolmogorov scale) is modeled using a validated drop model based on nonequilibrium evaporation and Stokes drag, with a drag coefficient accounting for a finite slip velocity between drops and gas, and for blowing from the drops. ${ }^{23}$ The application of this single drop model is totally consistent with the small volume fraction of the particles (see below), even in the developing regions of preferential concentration (although the assumption obviously deteriorates with increasing volume fraction). Drops and gas are entirely coupled, both dynamically and thermodynamically, and the effect of the droplets on the gas is modeled through source terms in the gas-phase equations. The present paper addresses the use of the DNS database of Miller and Bellan ${ }^{19}$ to evaluate SGS closures. Specifically, we examine three Reynolds numbers ( $\mathrm{Re}$, based on initial vorticity thickness, $\delta_{\omega, 0}$, and initial velocity difference, $\left.\Delta U_{0}\right)$ and mass loading (ML) combinations: $\operatorname{Re}=500$, $\mathrm{ML}=0.2$ (volume fraction of $\left.2.9 \times 10^{-4}\right) ; \mathrm{Re}=500, \mathrm{ML}=0.5$ (volume fraction of $7.2 \times 10^{-4}$ ); $\mathrm{Re}=600, \mathrm{ML}=0.2$. We consider the fully developed flow situation for all cases, corresponding to a dimensionless time $t \Delta U_{0} / \delta_{\omega, 0}$ of about 85.

\section{GOVERNING EQUATIONS}

The governing equations are formulated in an EulerianLagrangian manner whereby the carrier gas is modeled in an Eulerian frame whereas the drops are followed on their trajectory in a Lagrangian frame. These equations for the gas phase are recalled from Miller and Bellan ${ }^{20}$ to be

$$
\begin{aligned}
& \frac{\partial \rho}{\partial t}+\frac{\partial}{\partial x_{j}}\left[\rho u_{j}\right]=S_{\mathrm{I}}, \\
& \frac{\partial \rho u_{i}}{\partial t}+\frac{\partial}{\partial x_{j}}\left[\rho u_{i} u_{j}+P \delta_{i j}-\sigma_{i j}\right]=S_{\mathrm{II}, i}, \\
& \frac{\partial \rho E}{\partial t}+\frac{\partial}{\partial x_{j}}\left[(\rho E+P) u_{j}-\lambda \frac{\partial T}{\partial x_{j}}-u_{i} \sigma_{i j}\right]=S_{\mathrm{III}}, \\
& \frac{\partial \rho Y_{V}}{\partial t}+\frac{\partial}{\partial x_{j}}\left[\rho Y_{V} u_{j}-\rho \Gamma \frac{\partial Y_{V}}{\partial x_{j}}\right]=S_{\mathrm{I}}, \\
& \sigma_{i j}=\mu\left(\frac{\partial u_{i}}{\partial x_{j}}+\frac{\partial u_{j}}{\partial x_{i}}-\frac{2}{3} \frac{\partial u_{k}}{\partial x_{k}} \delta_{i j}\right), \\
& P=\rho R T, \\
& E=\frac{1}{2} u_{i} u_{i}+C_{v} T+h_{V}^{0} Y_{V}, \\
& R=Y_{V} R_{V}+\left(1-Y_{V}\right) R_{C}, \\
& C_{v}=Y_{V} C_{v, V}+\left(1-Y_{V}\right) C_{v, C}, \\
& C_{p}=Y_{V} C_{p, V}+\left(1-Y_{V}\right) C_{p, C}=R+C_{v},
\end{aligned}
$$

where $\rho$ is the gas phase density, $u_{i}$ is the gas phase velocity, $E=e+u_{i} u_{i} / 2$ is the total gas energy (internal $e$, plus kinetic), $h_{V}^{0}$ is a non-zero reference enthalpy of the evaporated species, ${ }^{20} P$ is the thermodynamic pressure, $\sigma_{i j}$ is the viscous stress tensor, $Y_{V}$ is the mass fraction of the evaporated species, subscript $V$ denotes the vapor, subscript $C$ denotes the carrier gas, the mass fraction of the carrier gas is $Y_{C}$ $=1-Y_{V}, \delta_{i j}$ is the Kronecker delta function, $R_{V}=R_{u} / W_{V}$ with $W$ denoting the molecular weight and $R_{u}$ being the universal gas constant, and $\mu, \lambda$, and $\Gamma$ are the constant gas phase viscosity, thermal conductivity, and Fickian diffusion coefficient, respectively. The right-hand-side terms $S_{\mathrm{I}}, S_{\mathrm{II}, i}$, and $S_{\mathrm{III}}$ describe the phase couplings of mass, momentum, and energy, respectively (discussed below). Note that a variable density (compressible) formulation is necessary even for low velocity flows due to the presence of both the mass source term and to nonequal molecular weight effects.

Coupled to the gas conservation equations, the Lagrangian particle equations for the position $\left(X_{i}\right)$, velocity $\left(v_{i}\right)$, temperature $\left(T_{d}\right)$, and mass $\left(m_{d}\right)$ are

$$
\begin{aligned}
& \frac{d X_{i}}{d t}=v_{i}, \\
& \frac{d v_{i}}{d t}=\frac{F_{i}}{m_{d}}, \\
& \frac{d T_{d}}{d t}=\frac{Q+\frac{d m_{d}}{d t} L_{v}}{m_{d} C_{L}},
\end{aligned}
$$

where the coupling between flow and drops is contained in the force term, $F_{i}$, the heat transfer term, $Q$, and the mass evolution from the drop term, $d m_{d} / d t$. Computation of the drag force $F_{i}$, the heat flux $Q$ and the evaporation rate $d m_{d} / d t$ requires knowledge of the gas phase variables $\left(u_{i}, T, Y_{V}, P\right)$ at the droplet locations, and involves in particular the use of validated models for the description of a single drop behavior. ${ }^{23,20}$ Employing these validated relationships, yields

$$
\begin{aligned}
& F_{i}=m_{d}\left(\frac{f_{1}}{\tau_{d}}\right)\left(u_{i}-v_{i}\right), \\
& Q=m_{d}\left(\frac{f_{2}}{\tau_{d}}\right)\left(\frac{N u C_{p, G}}{3 \operatorname{Pr}}\right)\left(T-T_{d}\right), \\
& \frac{d m_{d}}{d t}=\dot{m}_{d}=-m_{d}\left(\frac{1}{\tau_{d}}\right)\left(\frac{S h}{3 \mathrm{Sc}}\right) \ln \left[1+B_{M}\right],
\end{aligned}
$$

where the subscript $d$ denotes individual droplet conditions, the particle time constant for Stokes flow is $\tau_{d}$ $=\rho_{L} D^{2} /(18 \mu), D$ is the droplet diameter, $C_{L}$ is the heat capacity of the liquid and the latent heat of evaporation is $L_{V}$. The gas mixture heat capacity is calculated using a mass averaging, $C_{p, G}=\left(1-Y_{V}\right) C_{p, C}+Y_{V} C_{p, V}$ (evaluated at the droplet location) where $C_{p, C}$ and $C_{p, V}$ are the constant pressure heat capacities of the carrier gas and vapor, respectively $\left(C_{v, C}\right.$ and $C_{v, V}$ are the corresponding constant volume heat capacities). The gas phase Prandtl and Schmidt numbers are $\operatorname{Pr}=\mu C_{p, G} / \lambda$ and $\mathrm{Sc}=\mu /(\rho \Gamma)$, respectively. The evapora- 
tion rate is determined by the mass transfer number, $B_{M}$ $=\left(Y_{S}-Y_{V}\right) /\left(1-Y_{S}\right)$ (subscript $S$ denotes droplet surface conditions). To account for the effects of finite droplet slip Reynolds numbers, the semiempirical Ranz-Marshall correlations are utilized for the Nusselt $(\mathrm{Nu})$ and Sherwood (Sh) numbers, whereas $f_{1}$ is an empirical correlation accounting for the effects of both finite slip and evaporation to Stokes drag. ${ }^{23}$ The function $f_{2}=\beta /\left(e^{\beta}-1\right)$ is an analytical evaporative heat transfer correction, where the nondimensional evaporation parameter $\beta=-1.5 \operatorname{Pr} \tau_{d} \dot{m}_{d} / m_{d}$ is constant for droplets obeying the " $D^{2}$ law." The vapor surface mass fraction is calculated directly from the surface molar fraction $\left(\chi_{s}\right)$ which is obtained by equating the vapor and liquid fugacities at the surface (i.e., $\chi_{s} P=P_{\text {sat }}$ ) with the saturation pressure $\left(P_{\text {sat }}\right)$ provided by the Clausius-Clapeyron relation yielding:

$$
\begin{aligned}
Y_{S} & =\frac{\chi_{s}}{\chi_{s}+\left(1-\chi_{s}\right) W_{C} / W_{V}}, \\
\chi_{s} & =\frac{P_{\mathrm{atm}}}{P} \exp \left\{\frac{L_{V}}{R_{V}}\left(\frac{1}{T_{B, L}}-\frac{1}{T_{d}}\right)\right\},
\end{aligned}
$$

where $P_{\text {atm }}$ is the atmospheric pressure, $T_{B, L}$ is the liquid saturation temperature at $P_{\text {atm }}$ (i.e., the normal boiling temperature). As shown by Miller and Bellan, ${ }^{20}$ the latent heat must be a linear function of temperature for calorically perfect species: $L_{V}=h_{V}^{0}-\left(C_{L}-C_{p, V}\right) T_{d}$.

Equations (11)-(17) allow the computation of the Eqs. (1)-(4) source terms which are (see Miller and Bellan ${ }^{20}$ )

$$
\begin{aligned}
& S_{\mathrm{I}}=-\sum_{\alpha} \frac{w_{\alpha}}{V}\left[\frac{d m_{d}}{d t}\right]_{\alpha} \\
& S_{\mathrm{II}, i}=-\sum_{\alpha} \frac{w_{\alpha}}{V}\left[F_{i}+\frac{d m_{d}}{d t} v_{i}\right]_{\alpha} \\
& S_{\mathrm{III}}=-\sum_{\alpha} \frac{w_{\alpha}}{V}\left[F_{i} v_{i}+Q+\frac{d m_{d}}{d t}\left(\frac{1}{2} v_{i} v_{i}+C_{p, V} T_{d}+h_{V}^{0}\right)\right]_{\alpha}
\end{aligned}
$$

where the summation is over the $\alpha$ droplets within a discretization volume $V$ associated with each grid point, and the weights $w_{\alpha}$ distribute the droplet contribution among the nearest eight grid points; this weighting is necessary because the drop locations do not coincide in general to Eulerian grid points. On the other hand, Eqs. (11)-(16) require the knowledge of the gas flow variables at the drop locations; this is accomplished by using a fourth order Lagrange interpolation procedure. $^{20}$

As explained by Miller and Bellan, ${ }^{20}$ even for the smaller mass loadings used in the calculations, the source terms exhibit spatial "spottiness", resulting in artificial oscillations. Tong and Wang ${ }^{24}$ discuss this spottiness and circumvent the problem by representing the number density around each particle by a Gaussian distribution, thereby defining a continuous field through this local averaging while also conserving the number of particles. To mitigate this inherent spottiness in an Eulerian/Lagrangian treatment, Miller and Bellan $^{20}$ minimally smoothed the source terms with a con- servative operator. This operation is not a filtering, as one of a filter's characteristics is to truncate the high wave number terms, resulting in a nonconservative procedure.

The governing equations are integrated in time using a fourth-order Runge-Kutta explicit scheme, with eighth-order finite differences for all derivatives and fourth-order Lagrangian interpolation of gas-phase variables to droplet locations, as explained in detail in Miller and Bellan ${ }^{20}$ ). The numerical mesh is uniform in all directions with $\Delta x_{1} \simeq \Delta x_{2}$ $\simeq \Delta x_{3}$.

The initial conditions are similar to those of Moser and Rogers $^{25}$ and we refer the reader to Miller and Bellan ${ }^{20}$ for details. Basically, the configuration chosen is that of the temporally developing mixing layer where the streamwise $\left(x_{1}\right)$, cross stream $\left(x_{2}\right)$, and spanwise $\left(x_{3}\right)$ coordinates are defined, and the respective lengths are $L_{1}=4 \lambda_{1}, L_{2}=1.1 L_{1}$, and $L_{3}=4 \lambda_{3}$, where $\lambda_{1}$ and $\lambda_{3}$ are the forcing wavelengths in the $x_{1}$ and $x_{3}$ directions, $\lambda_{1}=7.29 \delta_{\omega, 0}$ (see Moser and Rogers $^{25}$ ) is the most unstable wavelength for the initial profile found from linear stability analysis, and $\lambda_{3}=0.6 \lambda_{1}$ following the suggestion of Ref. 25; for all the simulations performed by Miller and $\operatorname{Bellan}^{20} L_{1}=0.2 \mathrm{~m}$. The relative amplitudes of the forcing perturbations with respect to the circulations are $10 \%$ and $2.25 \%$ in the spanwise and streamwise directions, respectively. The boundary conditions used in conjunction with the conservation equations were periodic in the $x_{1}$ and $x_{3}$ directions, and adiabatic slip-wall conditions in the $x_{2}$ direction were employed. To insure physical consistency and avoid numerical instabilities, the wave decomposition method of Poinsot and Lele ${ }^{26}$ was used at the slipwall boundaries. The initial vorticity thickness was $\delta_{\omega, 0}$ and $\delta_{\omega}(t)=\Delta U_{0} /\left\langle\partial u_{1} / \partial x_{2}\right\rangle_{\max }$ where the brackets \langle\rangle indicate averaging over homogeneous $x_{1}-x_{3}$ planes. The freestream velocity difference across the layer, $\Delta U_{0}$ was calculated from a specified value of the convective Mach number, $M_{c}$.

The purpose of this investigation is to develop a SGS model to be utilized in a LES for a drop laden shear layer. Just as in the DNS, in the LES, the gas phase equations will be solved in an Eulerian frame, but at the LES, larger scale rather than the DNS scale; whereas the drops will be followed in a Lagrangian frame. Therefore, we can immediately identify two additional issues to that of the expected modeling of the SGS terms in the filtered gas phase equations. First, gas phase variables being calculated on the larger grid may no longer be a good representation of the equivalent DNS values, and the question arises on how to accurately calculate these unfiltered values at the drop locations. This calculation is important in two-phase flows because the source terms are affected by the values of the gas variables at the drop locations (i.e, temperature, velocity, and mass fractions); small errors in the calculation of these variables might translate into large errors in the source terms, according to the nonlinear dependencies. Moreover, since in a DNS/LES procedure the SGS models developed from the DNS at relatively low $\operatorname{Re}\left[\sim O\left(10^{3}\right)\right]$ are assumed to remain valid at much larger Re values characteristic of turbulence, it is likely that the accurate calculation of the unfiltered gas variables will be even more important when the SGS models are used at the higher Re. Indeed, for the relatively low $\mathrm{Re}$ of the 
DNS, the amount of energy in the subgrid scales is quite small, and thus the influence of the SGS model on the flow field in an LES will be correspondingly small; at larger Re these small errors might be greatly amplified. Second, the drop dependent variables are accurately calculated at each drop location if every single drop is followed on its trajectory. However, since the gas phase source terms in the filtered equations represent averages over the drops in the filtered volume, there is an inherent degree of uncertainty on how to model these source terms, i.e., how to accurately represent them using only information at the LES scale. We address in this paper the first issue identified above and defer the discussion of the second issue to a future publication.

\section{FILTERED GOVERNING EQUATIONS}

\section{A. General equations}

The general definition of a filter operation is

$$
\bar{\phi}(\vec{x})=\int_{V} \phi(\vec{y}) G_{\Delta}(\vec{x}-\vec{y}) d \vec{y},
$$

where $G_{\Delta}$ is the filter function, and $V$ is the filtering volume. In this study, we use a cubic top-hat filter, in which $V$ is a cube of sides $\Delta$, and $G_{\Delta}$ is simply a volume average:

$$
G_{\Delta}(\vec{x}-\vec{y})=\frac{1}{\Delta^{3}} \prod_{i=1}^{3}\left\{H\left(\Delta / 2-\left|x_{i}-y_{i}\right|\right)\right\},
$$

where $H(x)$ is the Heaviside function and $\Delta$ is the filter width. Our choice is guided by the simplicity of the associated integrations according to Eq. (21), and we disregard at this point the fact that the filtered equations are no longer invariant to coordinate rotations other than orthogonal. Since the goal of this investigation is to perform a LES of the shear layer using the SGS derived herein (and compare it with the filtered DNS), this lack of invariance can be tolerated. One of the properties of Eq. (21) is that for $\phi=1, \bar{\phi}=1$ as well. For compressible flow, we define the traditional Favre filtered variables as $\widetilde{\phi}=\overline{\rho \phi} / \bar{\rho}$, thereby removing the density fluctuations from the averaged equations, as well as all SGS terms that would be associated with them. Applying the filtering, and assuming that differentiation and filtering commute (this is true if a uniform filter is used, ${ }^{27}$ as is done here except within distance $\Delta / 2$ of the slipwall boundaries), the gas phase equations become

$$
\begin{aligned}
& \frac{\partial \bar{\rho}}{\partial t}+\frac{\partial}{\partial x_{j}}\left[\bar{\rho} \widetilde{u}_{j}\right]=\bar{S}_{I}, \\
& \frac{\partial \bar{\rho} \widetilde{u}_{i}}{\partial t}+\frac{\partial}{\partial x_{j}}\left[\bar{\rho} \widetilde{u}_{i} \widetilde{u}_{j}+\bar{P} \delta_{i j}-\widetilde{\sigma}_{i j}+\bar{\rho} \tau_{i j}\right]=\bar{S}_{\mathrm{II}, i}, \\
& \frac{\partial \bar{\rho} \widetilde{E}}{\partial t}+\frac{\partial}{\partial x_{j}}\left[(\bar{\rho} \widetilde{E}+\bar{P}) \tilde{u}_{j}-\lambda \frac{\partial \bar{T}}{\partial x_{j}}-\tilde{u}_{i} \widetilde{\sigma}_{i j}\right]+\frac{\partial}{\partial x_{j}}\left[\widetilde{u}_{i} \bar{\rho} \tau_{i j}\right. \\
& \left.\quad+C_{p, C} \bar{\rho} \theta_{j}+\left\{\left(C_{p, V}-C_{p, C}\right) \bar{T}+h_{V}^{0}\right\} \bar{\rho} \eta_{j}\right]=\bar{S}_{\mathrm{III}}, \\
& \frac{\partial \bar{\rho} \widetilde{Y}_{V}}{\partial t}+\frac{\partial}{\partial x_{j}}\left[\bar{\rho} \widetilde{Y}_{V} \widetilde{u}_{j}-\bar{\rho} \Gamma \frac{\partial \widetilde{Y}_{V}}{\partial x_{j}}+\bar{\rho} \eta_{j}\right]=\bar{S}_{\mathrm{I}},
\end{aligned}
$$

where we have defined

$$
\begin{aligned}
& \tau_{i j}=\widetilde{u_{i} u_{j}}-\widetilde{u}_{i} \tilde{u}_{j}, \\
& \theta_{j}=\widetilde{T u_{j}}-\bar{T} \widetilde{u}_{j}, \\
& \eta_{j}=\widetilde{Y_{V} u_{j}}-\widetilde{Y}_{V} \widetilde{u}_{j}, \\
& \widetilde{\sigma}_{i j}=\mu\left(\frac{\partial \widetilde{u}_{i}}{\partial x_{j}}+\frac{\partial \widetilde{u}_{j}}{\partial x_{i}}-\frac{2}{3} \frac{\partial \widetilde{u}_{k}}{\partial x_{k}} \delta_{i j}\right), \\
& \widetilde{C}_{p}=\widetilde{Y}_{V} C_{p, V}+\left(1-\widetilde{Y}_{V}\right) C_{p, C}, \\
& \widetilde{C}_{v}=\widetilde{Y}_{V} C_{v, V}+\left(1-\widetilde{Y}_{V}\right) C_{v, C}, \\
& \widetilde{R}=\widetilde{Y}_{V} R_{V}+\left(1-\widetilde{Y}_{V}\right) R_{C}=\widetilde{C}_{p}-\widetilde{C}_{v}
\end{aligned}
$$

and it has been assumed that

$$
\begin{aligned}
& \bar{\sigma}_{i j}=\widetilde{\sigma}_{i j}, \\
& \overline{u_{i} \sigma_{i j}}=\widetilde{u}_{i} \widetilde{\sigma}_{i j}, \\
& \frac{1}{2}\left(\overline{\rho u_{i} u_{i} u_{j}}-\overline{\rho u_{i} u_{i}} \widetilde{u}_{j}\right)=\bar{\rho} \tau_{i j} \widetilde{u}_{i}, \\
& \overline{\rho Y_{V} T}=\overline{\rho Y_{V}} \bar{T}, \\
& \overline{\rho Y_{V} T u_{j}}-\overline{\rho Y_{V} T} \widetilde{u}_{j}=\bar{\rho} \bar{T}\left(\widetilde{Y_{V} u_{j}}-\widetilde{Y}_{V} \widetilde{u}_{j}\right), \\
& \bar{T}=\widetilde{T}, \\
& \bar{P}=\bar{\rho} \widetilde{R} \bar{T}, \\
& \widetilde{E}=\frac{1}{2} \widetilde{u}_{i} \widetilde{u}_{i}+\widetilde{C}_{v} \bar{T}+h_{V}^{0} \widetilde{Y}_{V}+\frac{1}{2} \tau_{i i}, \\
& \frac{\partial Y_{V}}{\rho \Gamma \frac{\partial x_{j}}{\rho} \Gamma \frac{\partial \widetilde{Y}_{V}}{\partial x_{j}} .}
\end{aligned}
$$

Equation (36) is inspired by the RANS equations and the term $\left(C_{p, V}-C_{p, C}\right) \bar{\rho} \eta_{j} \bar{T}+C_{p, C} \bar{\rho} \theta_{j}$ is a direct result of the assumption of Eq. (38). For example, if we assume instead that

$$
\overline{\rho Y_{V} T u_{j}}-\overline{\rho Y_{V} T} \tilde{u}_{j}=\bar{\rho} \widetilde{Y}_{V}\left(\widetilde{T u_{j}}-\bar{T} \tilde{u}_{j}\right),
$$

this term would be replaced by $\widetilde{C}_{p} \bar{\rho} \theta_{j}$. Moreover, Eq. (42) is derived (for constant $\Gamma$ ) from

$$
\begin{aligned}
\overline{\rho \frac{\partial Y_{V}}{\partial x_{j}}} & =\frac{\overline{\partial \rho Y_{V}}}{\partial x_{j}}-\overline{Y_{V} \frac{\partial \rho}{\partial x_{j}}} \\
& =\frac{\partial \bar{\rho} \tilde{Y}_{V}}{\partial x_{j}}-\overline{Y_{V} \frac{\partial \rho}{\partial x_{j}}} \\
& =\bar{\rho} \frac{\partial \tilde{Y}_{V}}{\partial x_{j}}+\left(\tilde{Y}_{V} \frac{\partial \bar{\rho}}{\partial x_{j}}-\overline{Y_{V} \frac{\partial \rho}{\partial x_{j}}}\right)
\end{aligned}
$$

by assuming the last term to be negligible. Also, in contrast to the single phase situation where the filtered equation of state contains $\widetilde{T}$ only, in the two phase case $\widetilde{Y_{V} T}$ appears as well. In the next section we will assess the validity of all assumptions introduced by Eqs. (34) -(39) and (42) and show that they are justified for the three sets of results analyzed.

In the present two phase flow formulation, a SGS model must contain not only models for the subgrid stresses, $\tau_{i j}$, 
TABLE I. Summary of DNS database.

\begin{tabular}{lcccc}
\hline \hline Case & Re & ML & Grid & Time \\
\hline TP500a & 500 & 0.2 & $252 \times 276 \times 152$ & 85.0 \\
TP500b & 500 & 0.5 & $252 \times 276 \times 152$ & 85.0 \\
TP600 & 600 & 0.2 & $300 \times 332 \times 180$ & 86.2 \\
\hline \hline
\end{tabular}

but also models for the subgrid heat flux $\theta_{j}$ and the subgrid species flux $\eta_{j}$. Furthermore, since it is $\bar{T}$ rather than $\widetilde{T}$ that appears in the heat flux in the energy equation, this introduces the quandary as to which is the appropriate averaging of the temperature. In the SGS model presented below we opt to use $\bar{T}$, making therefore the assumption that $\bar{T}=\widetilde{T}$, and note that using $\widetilde{T}$ requires the assumption ${ }^{28}$ that $\partial \widetilde{T} / \partial x_{j}$ $=\partial \bar{T} / \partial x_{j}$; an assessment of the former assumption is presented below.

The SGS modeling is performed with the understanding that the droplet equations will still be solved in the LES, except that in LES the gas-phase variables $\left(u_{i}, T, Y_{V}, P\right)$ at the droplet locations are no longer immediately available and need to be derived from the filtered variables $\left(\widetilde{u}_{i}, \bar{T}, \widetilde{Y}_{V}, \bar{P}\right)$. Therefore, in order to be able to utilize a SGS model, one must also construct a model of each of the unfiltered gasphase variables as functions of the filtered values of the same variable. These models will be derived in the next section.

\section{B. Magnitude of terms and evaluation of underlying assumptions in the LES equations}

\section{Magnitude of terms in LES equations}

An evaluation of the magnitude of various terms in the LES equations is prerequisite not only to assessing the assumptions of Eqs. (34)-(39) and (42), but it is also crucial in providing both an understanding of the balance among terms, and an intuition on the models that may be used for SGS modeling. This evaluation is performed using the three sets of results from the database generated by Miller and Bellan, ${ }^{19}$ TP500a, TP500b and TP600 of a transitional, temporal drop laden mixing layer whose characteristics are summarized in Table I. As stated above, the Reynolds number,

TABLE II. Magnitude (root-mean-square) of terms in filtered $x_{1}$ momentum equation.

\begin{tabular}{cccc}
\hline \hline $\bar{\rho} \widetilde{u}_{1}$ equation $(i=1)$ & TP500a & TP500b & TP600 \\
\hline$\frac{\partial \bar{\rho} \widetilde{u}_{i} \widetilde{u}_{j}}{\partial x_{j}}$ & $3.9 \times 10^{5}$ & $4.0 \times 10^{5}$ & $4.4 \times 10^{5}$ \\
$\frac{\partial \bar{P} \delta_{i j}}{\partial x_{j}}$ & $3.4 \times 10^{5}$ & $3.4 \times 10^{5}$ & $3.5 \times 10^{5}$ \\
$\frac{\partial \bar{\rho} \tau_{i j}}{\partial x_{j}}$ & $7.1 \times 10^{4}$ & $7.9 \times 10^{4}$ & $7.1 \times 10^{4}$ \\
$\frac{\partial \widetilde{\sigma}_{i j}}{\partial x_{j}}$ & $1.5 \times 10^{4}$ & $1.7 \times 10^{4}$ & $2.0 \times 10^{4}$ \\
$\bar{S}_{\mathrm{II}, i}\left(\bar{\sigma}_{i j}-\widetilde{\sigma}_{i j}\right)$ & $9.4 \times 10^{2}$ & $1.6 \times 10^{3}$ & $1.1 \times 10^{3}$ \\
$\frac{\partial x_{j}}{2}$ & & $3.6 \times 10^{4}$ & $1.3 \times 10^{4}$ \\
\hline \hline
\end{tabular}

TABLE III. Magnitude (root-mean-square) of terms in filtered $x_{2}$ momentum equations.

\begin{tabular}{cccc}
\hline \hline $\bar{\rho} \widetilde{u}_{2}$ equation $(i=2)$ & TP500a & TP500b & TP600 \\
\hline$\frac{\partial \bar{\rho} \widetilde{u}_{i} \widetilde{u}_{j}}{\partial x_{j}}$ & $4.3 \times 10^{5}$ & $4.0 \times 10^{5}$ & $4.7 \times 10^{5}$ \\
$\frac{\partial \bar{P} \delta_{i j}}{\partial x_{j}}$ & $3.7 \times 10^{5}$ & $3.2 \times 10^{5}$ & $3.5 \times 10^{5}$ \\
$\frac{\partial \bar{\rho} \tau_{i j}}{\partial x_{j}}$ & $7.7 \times 10^{4}$ & $7.8 \times 10^{4}$ & $7.5 \times 10^{4}$ \\
$\frac{\partial \widetilde{\sigma}_{i j}}{\partial x_{j}}$ & $1.5 \times 10^{4}$ & $1.6 \times 10^{4}$ & $2.0 \times 10^{4}$ \\
$\bar{S}_{\mathrm{II}, i}$ & $1.1 \times 10^{4}$ & $2.7 \times 10^{4}$ & $1.1 \times 10^{4}$ \\
$\frac{\partial\left(\bar{\sigma}_{i j}-\widetilde{\sigma}_{i j}\right)}{\partial x_{j}}$ & $2.8 \times 10^{3}$ & $3.0 \times 10^{3}$ & $3.1 \times 10^{3}$ \\
\hline
\end{tabular}

$\mathrm{Re}$, is based on initial vorticity thickness, $\delta_{\omega, 0}$, and initial velocity difference, $\Delta U_{0}$. The mass loading, ML, is the initial loading in the droplet-laden stream and the database contains the results at the dimensionless time of $t \Delta U_{0} / \delta_{\omega, 0}$ $=85$. All three simulations were performed in a domain of size $0.2 \mathrm{~m} \times 0.22 \mathrm{~m} \times 0.12 \mathrm{~m}$. Our analysis is performed on the same grid as the DNS, with the resolutions listed in Table I, and all derivatives are calculated employing the same eighth-order finite-difference operator as in the DNS. In all cases, we use the cubic top-hat (box) filter of Eq. (22), with filter width $\Delta=4 \max \left(\Delta x_{1}, \Delta x_{2}, \Delta x_{3}\right)$, where $\Delta x_{1} \simeq \Delta x_{2}$ $\simeq \Delta x_{3}$.

Presented in Tables II-VI is such an evaluation for the momentum Eq. (24) (Tables II-IV), the energy Eq. (25) (Table V), and the species Eq. (26) (Table VI). Listed in the tables are the global root-mean-squares $[\operatorname{RMS}(\phi)$ $=\sqrt{\left\langle\left\langle\left\langle\phi^{2}\right\rangle\right\rangle\right\rangle}$, where $\langle\langle\langle\rangle\rangle\rangle$ denotes averaging over the entire domain] for cases TP500a, TP500b, and TP600. From Tables II-IV it is immediately apparent that in the three momentum equations $\partial \bar{\rho} \widetilde{u}_{i} \widetilde{u}_{j} / \partial x_{j}$ and $\partial \bar{P} / \partial x_{j}$ are the largest terms, and that they are of about the same order of magnitude. The smallest term, which is two orders of magnitude smaller than the largest, is $\partial\left(\bar{\sigma}_{i j}-\widetilde{\sigma}_{i j}\right) / \partial x_{j}$, justifying the

TABLE IV. Magnitude (root-mean-square) of terms in filtered $x_{3}$ momentum equation.

\begin{tabular}{cccc}
\hline \hline $\bar{\rho} \widetilde{u}_{3}$ equation $(i=3)$ & TP500a & TP500b & TP600 \\
\hline$\frac{\partial \bar{\rho} \widetilde{u}_{i} \widetilde{u}_{j}}{\partial x_{j}}$ & $3.8 \times 10^{5}$ & $3.7 \times 10^{5}$ & $4.5 \times 10^{5}$ \\
$\frac{\partial \bar{P} \delta_{i j}}{\partial x_{j}}$ & $2.8 \times 10^{5}$ & $2.8 \times 10^{5}$ & $3.1 \times 10^{5}$ \\
$\frac{\partial \bar{\rho} \tau_{i j}}{\partial x_{j}}$ & $5.8 \times 10^{4}$ & $6.5 \times 10^{4}$ & $6.7 \times 10^{4}$ \\
$\frac{\partial \widetilde{\sigma}_{i j}}{\partial x_{j}}$ & $1.4 \times 10^{4}$ & $1.4 \times 10^{4}$ & $1.9 \times 10^{4}$ \\
$\bar{S}_{\mathrm{II}, i}$ & & & \\
$\frac{\partial\left(\bar{\sigma}_{i j}-\widetilde{\sigma}_{i j}\right)}{\partial x_{j}}$ & $5.8 \times 10^{3}$ & $2.6 \times 10^{4}$ & $8.1 \times 10^{3}$ \\
\hline \hline
\end{tabular}


TABLE V. Magnitude (root-mean-square) of terms of filtered energy equation.

\begin{tabular}{cccc}
\hline \hline $\bar{\rho} \widetilde{E}$ equation & TP500a & TP500b & TP600 \\
\hline$\frac{\partial(\bar{\rho} \widetilde{E}+\bar{P}) \widetilde{u}_{j}}{\partial x_{j}}$ & $2.6 \times 10^{8}$ & $2.8 \times 10^{8}$ & $3.3 \times 10^{8}$ \\
$\frac{\partial\left[\overline{(\rho E+P) u_{j}}-(\bar{\rho} \widetilde{E}+\bar{P}) \widetilde{u}_{j}\right]}{\partial x_{j}}$ & $1.7 \times 10^{8}$ & $1.9 \times 10^{8}$ & $2.0 \times 10^{8}$ \\
$\bar{S}_{I I I}$ & $1.7 \times 10^{7}$ & $2.9 \times 10^{7}$ & $1.9 \times 10^{7}$ \\
$\frac{\partial}{\partial x_{j}}\left(\lambda \frac{\partial \bar{T}}{\partial x_{j}}\right)$ & $1.2 \times 10^{7}$ & $1.7 \times 10^{7}$ & $1.6 \times 10^{7}$ \\
$\frac{\partial \widetilde{u}_{i} \widetilde{\sigma}_{i j}}{\partial x_{j}}$ & $1.9 \times 10^{6}$ & $2.0 \times 10^{6}$ & $2.4 \times 10^{6}$ \\
$\frac{\partial u_{i} \sigma_{i j}}{\partial x_{j}}$ & $1.8 \times 10^{6}$ & $1.9 \times 10^{6}$ & $2.4 \times 10^{6}$ \\
$\frac{\partial\left(\overline{u_{i} \sigma_{i j}}-\widetilde{u}_{i} \widetilde{\sigma}_{i j}\right)}{\partial x_{j}}$ & $7.6 \times 10^{5}$ & $8.5 \times 10^{5}$ & $9.1 \times 10^{5}$ \\
\hline \hline
\end{tabular}

assumption $\bar{\sigma}_{i j}=\widetilde{\sigma}_{i j}$. In the middle range are $\partial \bar{\rho} \tau_{i j} / \partial x_{j}$ and $\partial \widetilde{\sigma}_{i j} / \partial x_{j}$, with the former term being about three times as large as the latter, whereas the source term $\bar{S}_{\mathrm{II}, i}$ is slightly smaller than the middle range terms. Note that the source terms which are listed in Tables II-VI are averaged over the whole grid, but are zero over about $20 \%$ of the grid where there are no droplets. However, planar RMS evaluations of the relative magnitude of the same terms in the center of the mixing layer (see Fig. 1 for case TP600) gives the same magnitude as that over the entire domain, thereby reinforcing our magnitude ranking above.

A similar evaluation of the energy equation terms displayed in Table $\mathrm{V}$ reveals that the inviscid subgrid term is of the same order of magnitude as the resolved inviscid term $\partial(\bar{\rho} \widetilde{E}+\bar{P}) \tilde{u}_{j} / \partial x_{j}$, followed by the heat flux and the resolved viscous term. The viscous subgrid term $\partial\left(\overline{u_{i} \sigma_{i j}}-\tilde{u}_{i} \widetilde{\sigma}_{i j}\right) / \partial x_{j}$ is about one-third of the viscous resolved term $\partial \widetilde{u}_{i} \widetilde{\sigma}_{i j} / \partial x_{j}$, suggesting that although $\partial \overline{u_{i} \sigma_{i j}} / \partial x_{j}$ and $\partial \widetilde{u}_{i} \widetilde{\sigma}_{i j} / \partial x_{j}$ are of

TABLE VI. Magnitude (root-mean-square) of terms of filtered species equation.

\begin{tabular}{cccc}
\hline \hline $\bar{\rho} \widetilde{Y}_{V}$ equation & TP500a & TP500b & TP600 \\
\hline$\frac{\partial \bar{\rho} \widetilde{Y}_{V} \widetilde{u}_{j}}{\partial x_{j}}$ & $1.5 \times 10^{2}$ & $1.8 \times 10^{2}$ & $2.0 \times 10^{2}$ \\
$\frac{\partial \bar{\rho} \eta_{i j}}{\partial x_{j}}$ & $2.8 \times 10^{1}$ & $3.4 \times 10^{1}$ & $2.8 \times 10^{1}$ \\
$\bar{S}_{\mathrm{I}}$ & $2.0 \times 10^{1}$ & $3.2 \times 10^{1}$ & $2.2 \times 10^{1}$ \\
$\frac{\partial}{\partial x_{j}}\left(\bar{\rho} \Gamma \frac{\partial \widetilde{Y}_{V}}{\partial x_{j}}\right)$ & $1.2 \times 10^{1}$ & $1.6 \times 10^{1}$ & $1.6 \times 10^{1}$ \\
$\frac{\partial}{\partial x_{j}}\left(\rho \Gamma \frac{\partial Y_{V}}{\partial x_{j}}\right)$ & $1.1 \times 10^{1}$ & $1.3 \times 10^{1}$ & $1.4 \times 10^{1}$ \\
$\frac{\partial}{\partial x_{j}}\left(\rho \Gamma \frac{\partial Y_{V}}{\partial x_{j}}\right)-\frac{\partial}{\partial x_{j}}\left(\bar{\rho} \Gamma \frac{\partial \widetilde{Y}_{V}}{\partial x_{j}}\right)$ & $6.2 \times 10^{0}$ & $1.1 \times 10^{1}$ & $7.8 \times 10^{0}$ \\
\hline \hline
\end{tabular}

the same magnitude, they are not perfectly correlated. The correlation between two terms is defined either by averaging over homogeneous $\left(x_{1}-x_{3}\right)$ planes

$$
\mathcal{R}\left(X, Y ; x_{2}\right)=\frac{\langle X Y\rangle}{\sqrt{\left\langle X^{2}\right\rangle} \sqrt{\left\langle Y^{2}\right\rangle}}
$$

or over the entire domain

$$
\mathcal{R}(X, Y)=\frac{\langle\langle\langle X Y\rangle\rangle\rangle}{\sqrt{\left\langle\left\langle\left\langle X^{2}\right\rangle\right\rangle\right\rangle\left\langle\left\langle\left\langle Y^{2}\right\rangle\right\rangle\right\rangle}},
$$

where by definition $\mathcal{R}$ is between -1 and 1 . As usual, values near 1 indicate strong positive correlation, values near -1 indicate strong negative correlation, whereas values near 0 indicate poor correlation. More precisely, we find that the correlation of $\partial \overline{u_{i} \sigma_{i j}} / \partial x_{j}$ and $\partial \tilde{u}_{i} \widetilde{\sigma}_{i j} / \partial x_{j}$ over the entire grid is 0.92, and a corresponding linear fit is $\partial \overline{u_{i} \sigma_{i j}} / \partial x_{j}$ $=0.95 \partial \widetilde{u}_{i} \widetilde{\sigma}_{i j} / \partial x_{j}$. As will be seen below, this correlation is only slightly worse than that between $\overline{u_{i} \sigma_{i j}}$ and $\tilde{u}_{i} \widetilde{\sigma}_{i j}$. Moreover, since $\partial\left(\overline{u_{i} \sigma_{i j}}-\widetilde{u}_{i} \widetilde{\sigma}_{i j}\right) / \partial x_{j}$ is the smallest term in the energy equation, it is not unreasonable to neglect it. The source term $\bar{S}_{\text {III }}$ is about the same size as the heat flux term justifying our expectation that it is an important term.

In Table VI we finally display a comparison of the magnitude of the species equation terms. Clearly, the subgrid term, the source term and the diffusion term are all of the same order, being an order of magnitude smaller than the resolved convective term. The smallest term $\partial\left(\overline{\rho \Gamma \partial Y_{V} / \partial x_{j}}\right) \partial x_{j}-\partial\left(\bar{\rho} \Gamma \partial \widetilde{Y}_{V} / \partial x_{j}\right) / \partial x_{j}$ is about half of $\partial\left(\bar{\rho} \Gamma \partial \widetilde{Y}_{V} / \partial x_{j}\right) / \partial x_{j}$, but could possibly be neglected if the correlation between $\overline{\rho \partial Y_{V} / \partial x_{j}}$ and $\bar{\rho} \partial \widetilde{Y}_{V} / \partial x_{j}$ is high; this is indeed the case, as discussed below.

Additional to the global RMS values shown in the tables, the planar RMS values of the various terms in each equation are presented for case TP600 in Fig. 1 (in the figures [ ] and \{\} are used to denote regular and Favre filtering, respectively), and they confirm the conclusions reached from the global RMS values.

\section{Evaluation of LES assumptions}

A systematic evaluation of the key assumptions made in deriving the LES equations is presented in Figs. 2-6 by showing the $\left(x_{1}-x_{3}\right)$ plane averages of the approximated terms. The analysis is carried out for case TP600 with $\Delta$ $=4 \max \left(\Delta x_{1}, \Delta x_{2}, \Delta x_{3}\right)$, as above. Clearly, the assumption of Eq. (36) $\left[\frac{1}{2}\left(\overline{\rho u_{i} u_{i} u_{j}}-\overline{\rho u_{i} u_{i}} \widetilde{u_{j}}\right)=\bar{\rho} \tau_{i j} \widetilde{u}_{i}\right]$ is quite reasonable, as shown in Fig. 2. The assumptions of Eqs. (39) $(\bar{T}=\widetilde{T})$ and (37) $\left(\overline{\rho Y_{V} T}=\overline{\rho Y_{V}} \bar{T}\right)$ are also justified for this flow, as seen in Fig. 3, although since the turbulent temperature fluctuations in this study appear to be quite small (as will be discussed below), these two assumptions may hold only in the range of parameters covered by this study, and caution is recommended in using them without justification in more general situations.

Figure 4 addresses the modeling of $\overline{\rho Y_{V} T u_{j}}$ through 

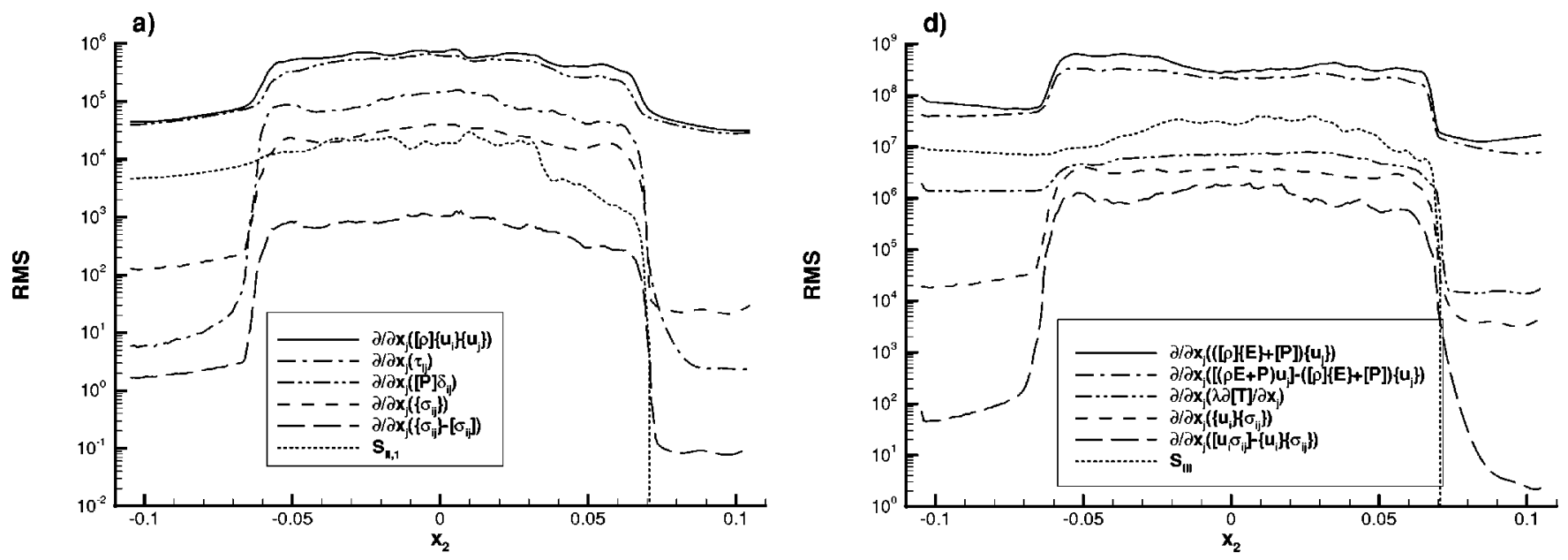

b)
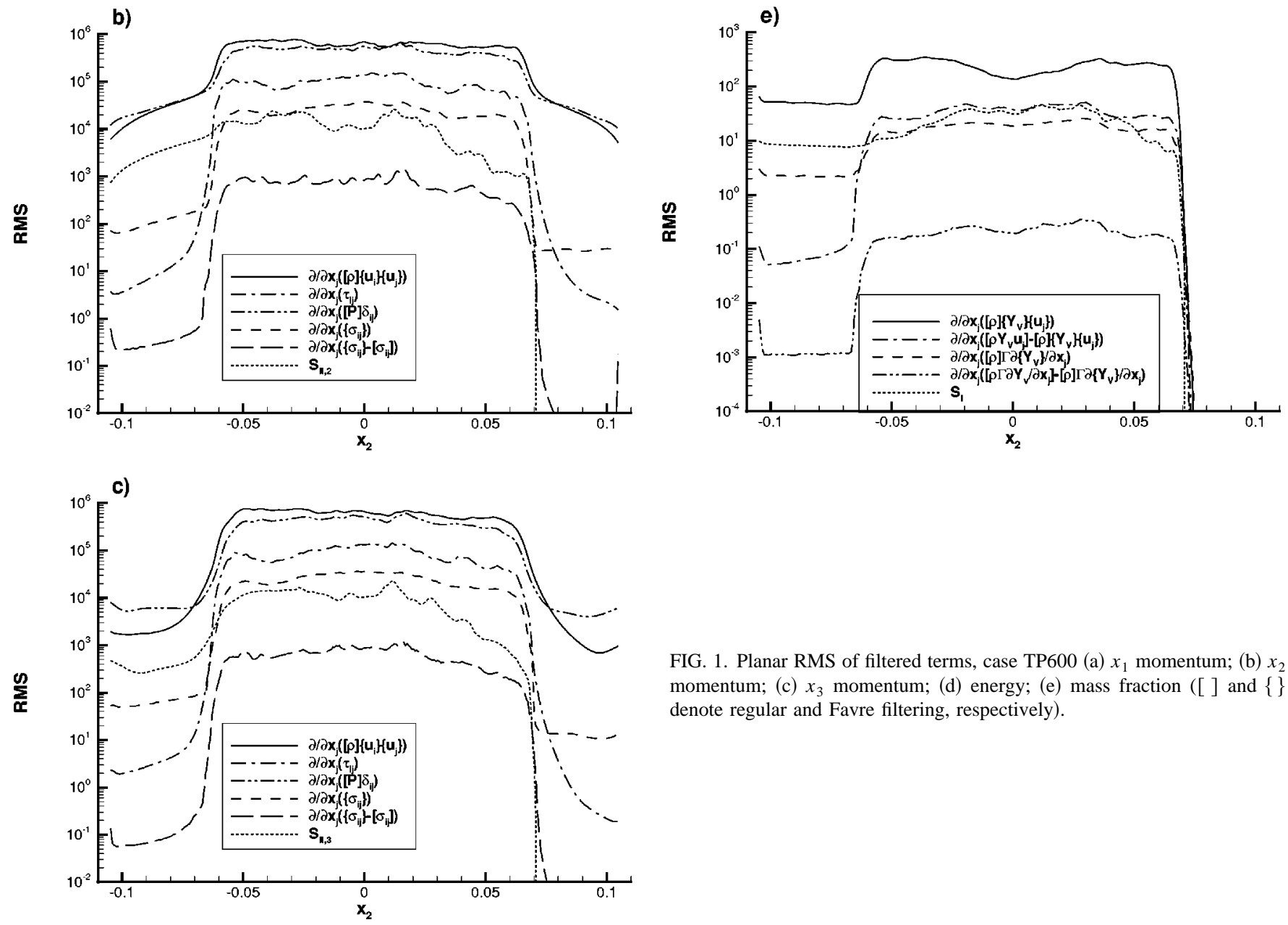

FIG. 1. Planar RMS of filtered terms, case TP600 (a) $x_{1}$ momentum; (b) $x_{2}$ momentum; (c) $x_{3}$ momentum; (d) energy; (e) mass fraction ([ ] and \{\} denote regular and Favre filtering, respectively).

either Eq. (38) or Eq. (43). As depicted in the figure, the right-hand side of Eq. (43), $\bar{\rho} \widetilde{Y}_{V}\left(\widetilde{T u_{j}}-\bar{T} \tilde{u}_{j}\right)$ is almost null and is additionally much smaller than the left-hand side $\overline{\rho Y_{V} T u_{j}}-\overline{\rho Y_{V} T} \tilde{u}_{j}$. However, Fig. 4 shows that the left-hand

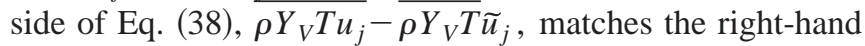
side, $\bar{\rho} \bar{T}\left(\widetilde{Y_{V} u_{j}}-\widetilde{Y}_{V} \widetilde{u}_{j}\right)$, thereby indicating that this assumption is correct. Moreover, the correlation over the entire grid for the two-sides of Eq. (43) is -0.270 , whereas it is 0.999 for Eq. (38), thus justifying the latter assumption.
To evaluate the assumption of Eq. (35), $\overline{u_{i} \sigma_{i j}}=\widetilde{u}_{i} \widetilde{\sigma}_{i j}$, we display in Fig. 5 the plane averages, $\overline{u_{i} \sigma_{i j}}-\widetilde{u}_{i} \widetilde{\sigma}_{i j}$ and $\tilde{u}_{i} \widetilde{\sigma}_{i j}$. Clearly, $\overline{u_{i} \sigma_{i j}}-\tilde{u}_{i} \widetilde{\sigma}_{i j}$ is small compared to $\tilde{u}_{i} \widetilde{\sigma}_{i j}$ and moreover a linear fit shows $\overline{u_{i} \sigma_{i j}}=0.94 \tilde{u}_{i} \widetilde{\sigma}_{i j}$, with a correlation of 0.97 ; thus the assumption $\overline{u_{i} \sigma_{i j}}=\widetilde{u}_{i} \widetilde{\sigma}_{i j}$ is justified.

Finally, to assess the assumption of Eq. (42), we display in Fig. 6 both $\overline{\rho \partial Y_{V} / \partial x_{j}}$ and $\bar{\rho} \partial \widetilde{Y}_{V} / \partial x_{j}$ and show them to be well correlated. Furthermore, a calculated linear fit shows $\overline{\rho \partial Y_{V} / \partial x_{j}}=0.997 \bar{\rho} \partial \widetilde{Y}_{V} / \partial x_{j}$ with a correlation of 0.996 , 

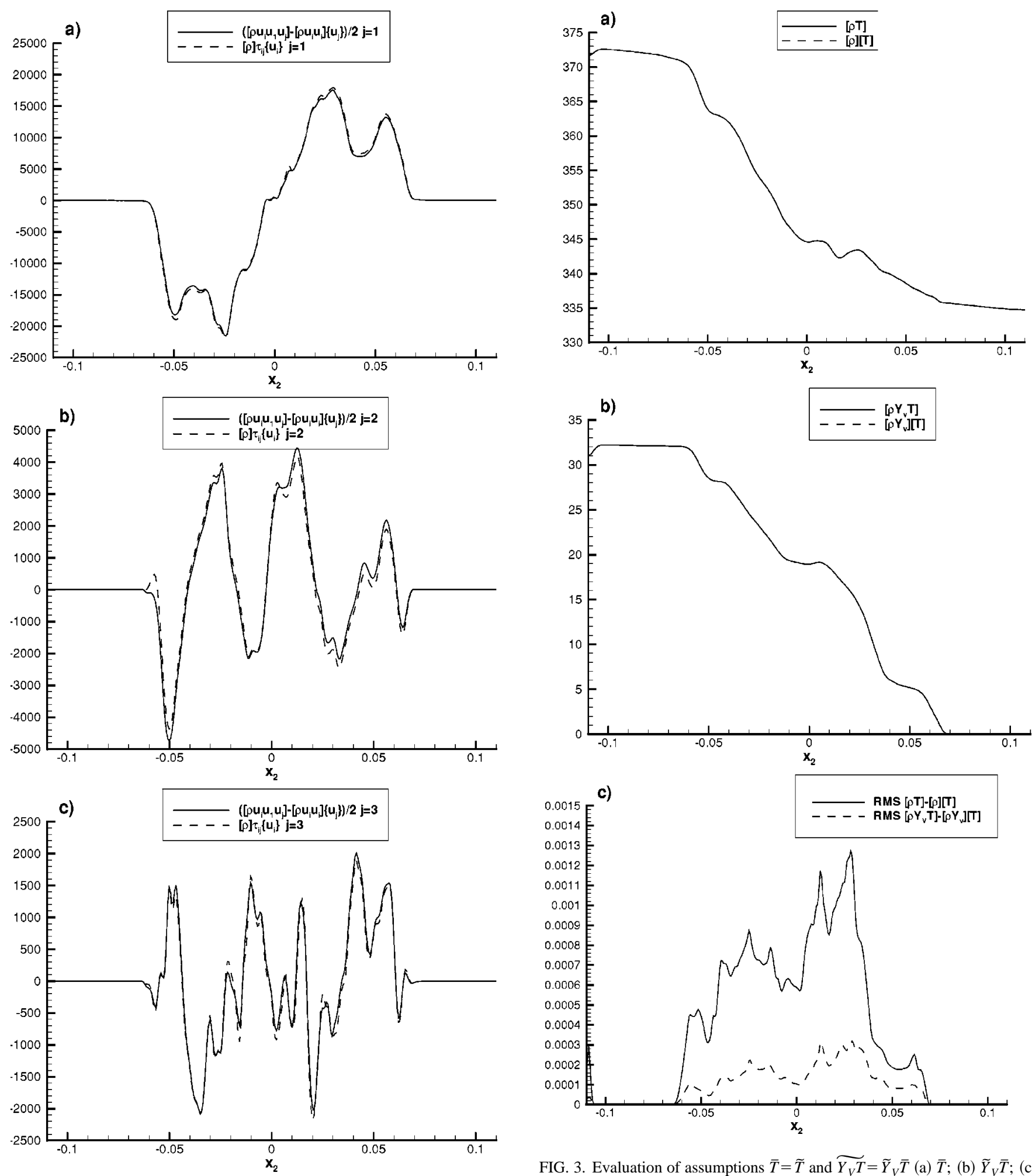

FIG. 2. Evaluation of assumption $\overline{\rho u_{i} u_{i} u_{j}}-\overline{\rho u_{i} u_{i}} \widetilde{u}_{j}=\bar{\rho} \tau_{i j} \widetilde{u}_{i}$ (a) $j=1$; (b) $j=2$; (c) $j=3$ ([ ] and \{\} denote regular and Favre filtering, respectively).

FIG. 3. Evaluation of assumptions $\bar{T}=\widetilde{T}$ and $\widetilde{Y_{V} T}=\widetilde{Y}_{V} \bar{T}$ (a) $\bar{T}$; (b) $\widetilde{Y}_{V} \bar{T}$; (c) RMS of differences ([ ] and \{\} denote regular and Favre filtering, respectively).

thereby confirming the intuitive idea that $\overline{\rho \partial Y_{V} / \partial x_{j}}$ and $\bar{\rho} \partial \widetilde{Y}_{V} / \partial x_{j}$ may be assumed equal.

\section{MODEL FOR UNFILTERED GAS PHASE VARIABLES}

As discussed above, the knowledge of the unfiltered gas phase variables is necessary at the droplet locations for the

calculation of the source terms in the conservation equations. However, these unfiltered gas variables are not available from the LES whose solution is obtained at the larger scale and at grid points rather than drop locations. The recovery of unfiltered variables from the filtered flow field, also known as deconvolution (as filtering is a mathematical convolution), has already been explored for generating subgrid scale mod- 

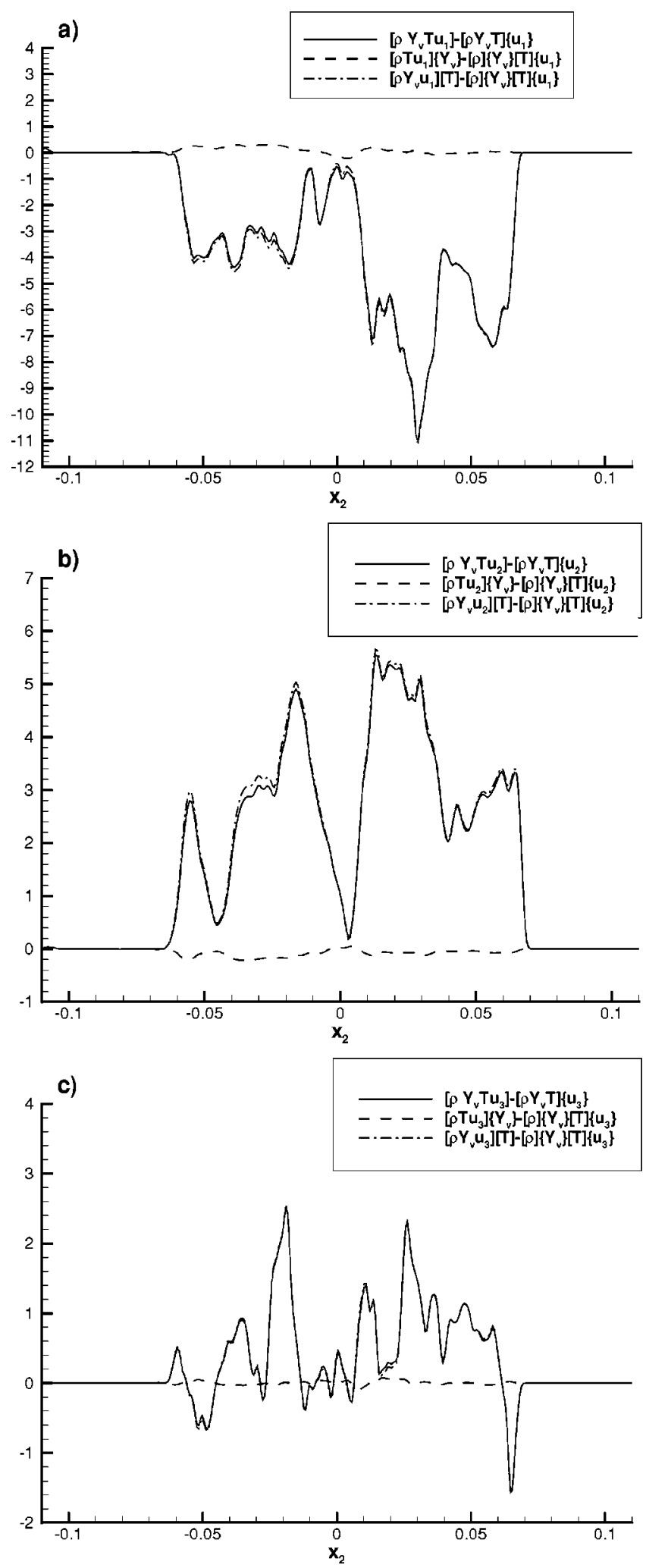

FIG. 4. Evaluation of assumptions regarding $\overline{\rho Y_{V} T u_{j}}-\overline{\rho Y_{V} T} \tilde{u}_{j}$ (a) $j=1$; (b) $j=2$; (c) $j=3$ ([ ] and \{\} denote regular and Favre filtering, respectively).

els in Refs. 29-31. Theoretically, the unfiltered variable can be found by applying the inverse of the filter function, i.e.,

$$
\phi(\vec{x})=\int_{V} \bar{\phi}(\vec{y}) G_{\Delta}^{-1}(\vec{x}-\vec{y}) d \vec{y},
$$
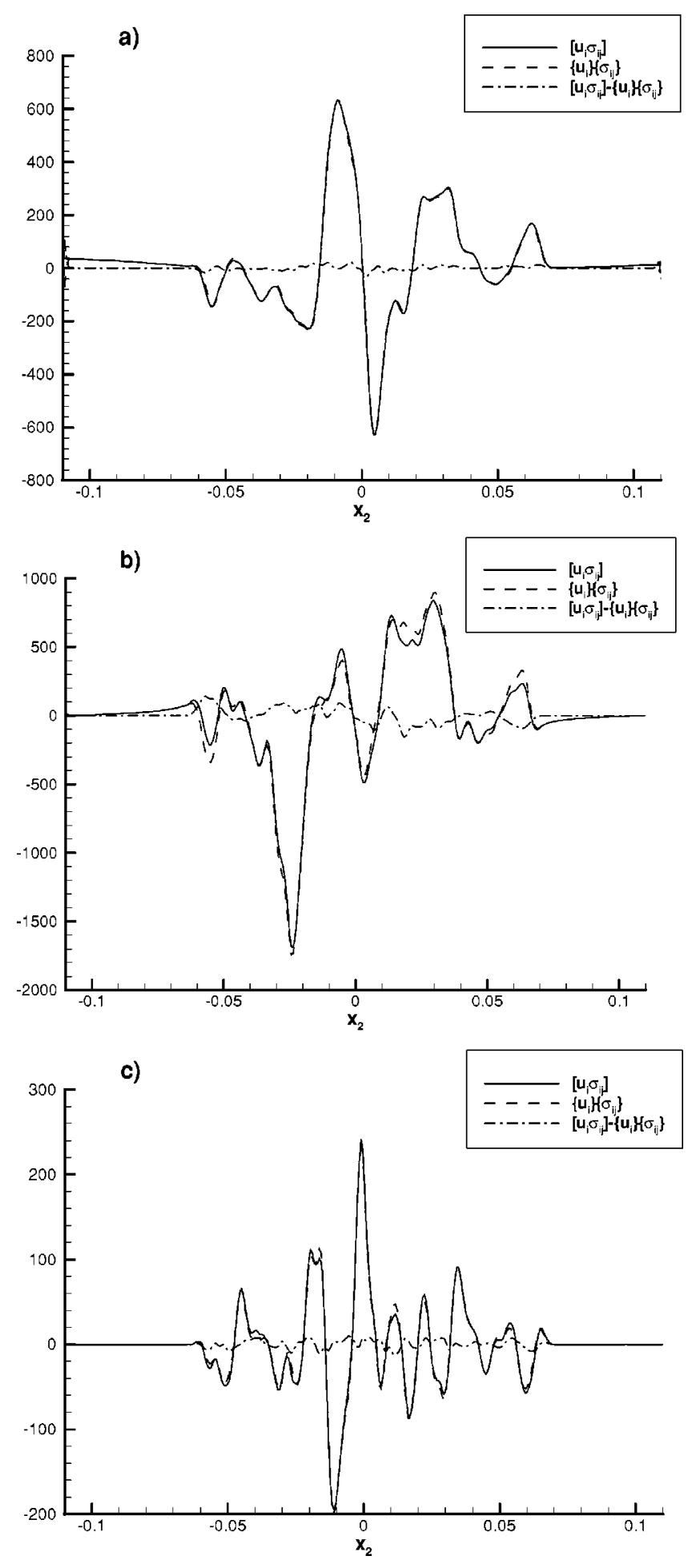

FIG. 5. Evaluation of assumption $\overline{u_{i} \sigma_{i j}}=\widetilde{u}_{i} \widetilde{\sigma}_{i j}$ (a) $j=1$; (b) $j=2$; (c) $j$ $=3$ ( [] and \{\} denote regular and Favre filtering, respectively).

or its numerical equivalent. In practice, such an inversion is not feasible because: (1) In many LES, particularly finite difference and finite volume computations, the filter is implicit and thus its inverse is difficult to define. (2) Filtering by definition discards high wave number information (since it has a smoothing effect) that in general cannot be recovered. (3) Discretization errors intervene as $\bar{\phi}$ is usually computed on the coarsest possible grid, which is generally too 

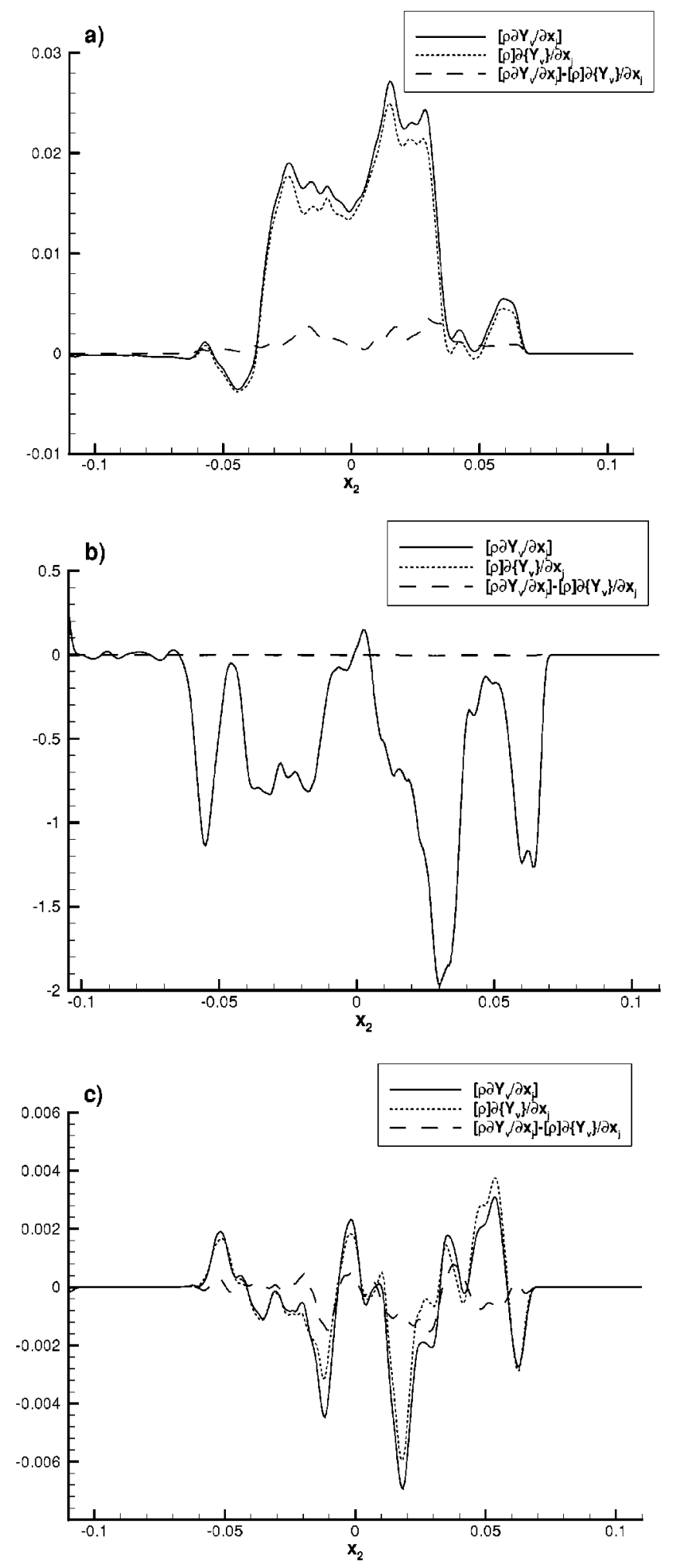

FIG. 6. Evaluation of assumption $\overline{\rho \partial Y_{V} / \partial x_{j}}=\bar{\rho} \partial \widetilde{Y}_{V} / \partial x_{j}$ (a) $j=1$; (b) $j$ $=2$; (c) $j=3$ ([ ] and \{\} denote regular and Favre filtering, respectively).

coarse to adequately represent $\phi$. This leads to decreased computational effort for LES compared to DNS, but further complicates the deconvolution. (4) The relation between $\phi$ and $\bar{\phi}$ is not a one-to-one mapping, since several realizations of $\phi$ on a grid can lead to the same $\bar{\phi}$. (5) Even if an inverse can be precisely defined, the computational cost of computing it may be prohibitive.
Hence, rather than seeking the inverse filtering operator, we seek an appropriate model for $\phi$, which we denote $\phi_{m}$, that captures the essential statistical properties of $\phi$ from its computed filtered form $\bar{\phi}$, where the bar is here a generic averaging denoting Favre filtering for $u_{i}$ and $Y_{V}$, and regular filtering for $T$ and $P$. The desired properties depend on the intent for using $\phi_{m}$; in the present study, it is to be interpolated to droplet locations to emulate droplet interaction with the flow field represented by $\phi$. Of necessity, the procedure used to generate $\phi_{m}$, i.e., the relation between $\phi$ and $\bar{\phi}$, depends on the filter used to generate $\bar{\phi}$. In effect, we must analyze the DNS database to reconstruct the information that was removed from the flow field upon filtering. We restrict our consideration to the top-hat filter Eq. (22), which can alternatively be viewed as a volume-averaging operation. We define by "appropriate model" a relationship that gives the value of the unfiltered variables as close in magnitude as possible to those of the DNS, and certainly better than one would obtain by simply interpolating the values of the filtered variables at the droplet locations. To guide the modeling, we will first consider the known DNS generic variable $\phi$ and $\bar{\phi}$. By definition, the standard deviation is

$$
\sigma=\sqrt{\phi^{\prime} \phi^{\prime}}=\sqrt{(\phi-\bar{\phi})^{2}}
$$

where $\phi^{\prime}$ is the fluctuating part of $\phi$, and thus the relation between $\phi$ and $\bar{\phi}$ is

$$
\phi=\bar{\phi}+f \sigma,
$$

where from the definition of $\sigma, f= \pm 1$. Intuitively, in this formulation, $f \sigma$ can be regarded as a correction to $\bar{\phi}$ with sign $f$ and magnitude $\sigma$. The goal of the modeling is to compute, from the filtered flow field, a model of $f \sigma, f_{m} \sigma_{m}$, that provides a better approximation to $\phi$ than does $f_{m} \sigma_{m}$ $=0$.

Because of the inherent simplicity, it is at first tempting to assume that $f$ randomly takes on values of -1 and 1 . However, one can show that this is not necessarily the case. If the filtering operation is viewed as a volume average, a relation between $\phi$ and $\bar{\phi}$ can be derived by considering the third-order Taylor expansion of $\phi$ integrated over the filtering volume $V$ of characteristic length $\Delta$ with centroid at $x_{0}$ $=\left(x_{1_{0}}, x_{2_{0}}, x_{3_{0}}\right)$ :

$\bar{\phi}\left(x_{0}\right)=\frac{1}{V} \int_{V} \phi(x) d V$,

$$
\begin{aligned}
\phi(x)= & \phi\left(x_{0}\right)+\frac{\partial \phi}{\partial x_{i}}\left(x_{0}\right)\left(x_{i}-x_{i_{0}}\right)+\frac{\partial^{2} \phi}{\partial x_{i} \partial x_{j}}\left(x_{0}\right) \frac{1}{2}\left(x_{i}-x_{i_{0}}\right) \\
& \times\left(x_{j}-x_{j_{0}}\right)+O\left(\Delta^{3}\right),
\end{aligned}
$$

where from the definition of the centroid, $(1 / V) \int_{V}\left(x_{i}\right.$ $\left.-x_{i_{0}}\right) d V=0$. If $V$ is symmetric, then

$$
\frac{1}{V} \int_{V}\left(x_{i}-x_{i_{0}}\right)\left(x_{j}-x_{j_{0}}\right) d V=0, \quad i \neq j
$$

and 


$$
I_{c} \Delta^{2}=\frac{1}{V} \int_{V}\left(x_{i}-x_{i_{0}}\right)\left(x_{j}-x_{j_{0}}\right) d V, \quad i=j
$$

is the (positive) moment of inertia, so that one may rewrite Eq. (50) as

$$
\bar{\phi}=\phi+\nabla^{2} \phi \frac{I_{c} \Delta^{2}}{2}+O\left(\Delta^{4}\right),
$$

where terms of $O\left(\Delta^{3}\right)$ vanish due to the symmetry of the filtering volume.

A comparison between Eqs. (49) and (54), and the interpretation that $f$ is a sign correction leads to the conclusion that $f$ will generally be $-\operatorname{sign}\left(\nabla^{2} \phi\right)$. However, $\phi$ is not available in a LES formulation, and from the available filtered quantities we can compute $\nabla^{2} \bar{\phi}$ rather than $\nabla^{2} \phi$. Therefore, to model $f$ we assume that $\nabla^{2} \bar{\phi}$ and $\nabla^{2} \phi$ have the same sign and evaluate this assumption for the gas phase variables in the section devoted to finalizing the SGS models.

To model $\sigma$, we note that the gas phase SGS terms which must be modeled have the generic form $\overline{\phi \phi}-\bar{\phi} \bar{\phi}$. If one defines $\sigma_{\mathrm{SGS}}$ as the SGS standard deviation,

$$
\sigma_{\mathrm{SGS}}=\sqrt{\overline{\overline{\phi \phi}}-\bar{\phi} \bar{\phi}}
$$

the relationship between $\sigma$ and $\sigma_{\mathrm{SGS}}$ can be elucidated by considering $\overline{\sigma^{2}}$

$$
\overline{\sigma^{2}}=\overline{(\phi-\bar{\phi})^{2}}=\overline{\phi \phi}-2 \overline{\phi \bar{\phi}}+\overline{\bar{\phi} \bar{\phi}}
$$

where the second filter is unweighted (e.g., regular rather than Favre) for all variables. To evaluate the terms in Eq. (56), we note that $\overline{\phi \phi}=\sigma_{\text {SGS }}^{2}+\bar{\phi} \bar{\phi}$ and that $\overline{\phi \bar{\phi}}$ can be written in terms of the local correlation between $\phi$ and $\bar{\phi}$

$$
\mathcal{R}(\phi, \bar{\phi})=\frac{\overline{\phi \bar{\phi}}}{\sqrt{\overline{\phi \phi}} \sqrt{\overline{\bar{\phi} \bar{\phi}}}} .
$$

If we assume that $\mathcal{R}(\phi, \bar{\phi}) \simeq 1$, then

$$
\overline{\sigma^{2}}=(\sqrt{\overline{\overline{\phi \phi}}}-\sqrt{\overline{\bar{\phi} \bar{\phi}}})^{2}=\left(\sqrt{\sigma_{\mathrm{SGS}}^{2}+\bar{\phi} \bar{\phi}}-\sqrt{\overline{\bar{\phi} \bar{\phi}}}\right)^{2} .
$$

The assumption that $\mathcal{R}(\phi, \bar{\phi}) \simeq 1$ was checked for all gas phase variables using the TP600 set of results and was found to be justified (see the finalization of the SGS models section). It is noteworthy that when using even small departures from the unity (i.e., $\mathcal{R}(\phi, \bar{\phi})=0.99)$, one obtains models for the unfiltered variables (in particular $Y_{V}$ ) that are considerably less accurate than those obtained using $\mathcal{R}(\phi, \bar{\phi}) \simeq 1$.

Defining $\bar{\sigma}=\sqrt{\overline{\sigma^{2}}}$, and using $\bar{\sigma}$ as a model for $\sigma$, we arrive at a model for $\phi$ of the form

$$
\phi=\bar{\phi}-\operatorname{sign}\left(\nabla^{2} \bar{\phi}\right) \bar{\sigma}
$$

i.e., $f_{m}=-\operatorname{sign}\left(\nabla^{2} \bar{\phi}\right), \sigma_{m}=\bar{\sigma}$.

\section{MODELS FOR SUBGRID CROSS-TERMS}

The success of the LES formalism depends considerably upon the fidelity of $\tau_{i j}=\widetilde{u_{i} u_{j}}-\tilde{u}_{i} \tilde{u}_{j}, \quad \theta_{j}=\widetilde{T u_{j}}-\bar{T} \tilde{u}_{j}, \quad \eta_{j}$ $=\widetilde{Y_{V} u_{j}}-\widetilde{Y_{V} \widetilde{u}_{j}}$, and $\sigma_{\mathrm{SGS}}^{2}=\overline{\phi \phi}-\bar{\phi} \bar{\phi}$ models in portraying the true magnitude of the unfiltered terms using the filtered variables. For LES in the gas-phase, models are required for the subgrid stresses $\tau_{i j}$, heat fluxes $\theta_{j}$, and species fluxes $\eta_{j}$; for the droplet description, models are required for the subgrid variances $\sigma_{\mathrm{SGS}}^{2}: \widetilde{u_{1} u_{1}}-\widetilde{u}_{1} \tilde{u}_{1}, \widetilde{u_{2} u_{2}}-\widetilde{u}_{2} \tilde{u}_{2}, \widetilde{u_{3} u_{3}}$ $-\tilde{u}_{3} \tilde{u}_{3}, \overline{T T}-\bar{T} \bar{T}, \widetilde{Y}_{V} Y_{V}-\tilde{Y}_{V} \tilde{Y}_{V}$, and $\overline{P P}-\bar{P} \bar{P}$. Since the subgrid stresses, the heat flux, the species flux and the subgrid variances are all of the same form, it seems reasonable and consistent to use the same type of model for all of them. In the following, we consider the possibility of subgrid modeling employing three different models: the traditional (e.g., constant coefficient) Smagorinsky model, the gradient model, and the scale-similarity model. ${ }^{6}$ We first define these three models below and introduce in each of the models a constant of proportionality that we further determine from comparisons of the modeled terms with the exact values of the subgrid terms as calculated from the unfiltered variables in the DNS database.

\section{A. Smagorinsky SGS model}

In the traditional Smagorinsky model, $\tau_{i j}, \theta_{j}$, and $\eta_{j}$ are expressed as (see Ref. 32)

$$
\begin{aligned}
& \tau_{i j}-\frac{1}{3} \tau_{k k}=-2 C_{R} \Delta^{2} \sqrt{\widetilde{S}_{k l} \widetilde{S}_{k l}}\left(\widetilde{S}_{i j}-\frac{1}{3} \widetilde{S}_{k k} \delta_{i j}\right), \\
& \theta_{j}=-\frac{C_{R} \Delta^{2}}{\operatorname{Pr}} \sqrt{\widetilde{S}_{k l} \widetilde{S}_{k l}} \frac{\partial \bar{T}}{\partial x_{j}}, \\
& \eta_{j}=-C_{R} \Delta^{2} \sqrt{\widetilde{S}_{k l} \widetilde{S}_{k l}} \frac{\partial \widetilde{Y}_{V}}{\partial x_{j}},
\end{aligned}
$$

where $C_{R}$ is a model constant and $\Delta$ is a filter width $\left(\tau_{k k}\right.$ can be neglected for small Mach number based on the velocity fluctuation $^{28}$ ). In Eqs. (60)-(62) the rate-of-strain tensor for the filtered velocities is defined as

$$
\widetilde{S}_{i j}=\frac{1}{2}\left(\frac{\partial \tilde{u}_{i}}{\partial x_{j}}+\frac{\partial \tilde{u}_{j}}{\partial x_{i}}\right) .
$$

We note that this form is the basis for most SGS models, concerned only with flow dynamics, but that it cannot be easily extended to compute the subgrid variances for $T, P$, and $Y_{V}$.

\section{B. Gradient SGS model}

In contrast to the traditional Smagorinsky model, the gradient model (e.g., Liu et al. $^{33}$ ) defined by

$$
\begin{gathered}
\tau_{i j}=C_{G} \Delta^{2} \frac{\partial \tilde{u}_{i}}{\partial x_{k}} \frac{\partial \tilde{u}_{j}}{\partial x_{k}}, \\
\theta_{j}=C_{G} \Delta^{2} \frac{\partial \bar{T}}{\partial x_{k}} \frac{\partial \tilde{u}_{j}}{\partial x_{k}},
\end{gathered}
$$



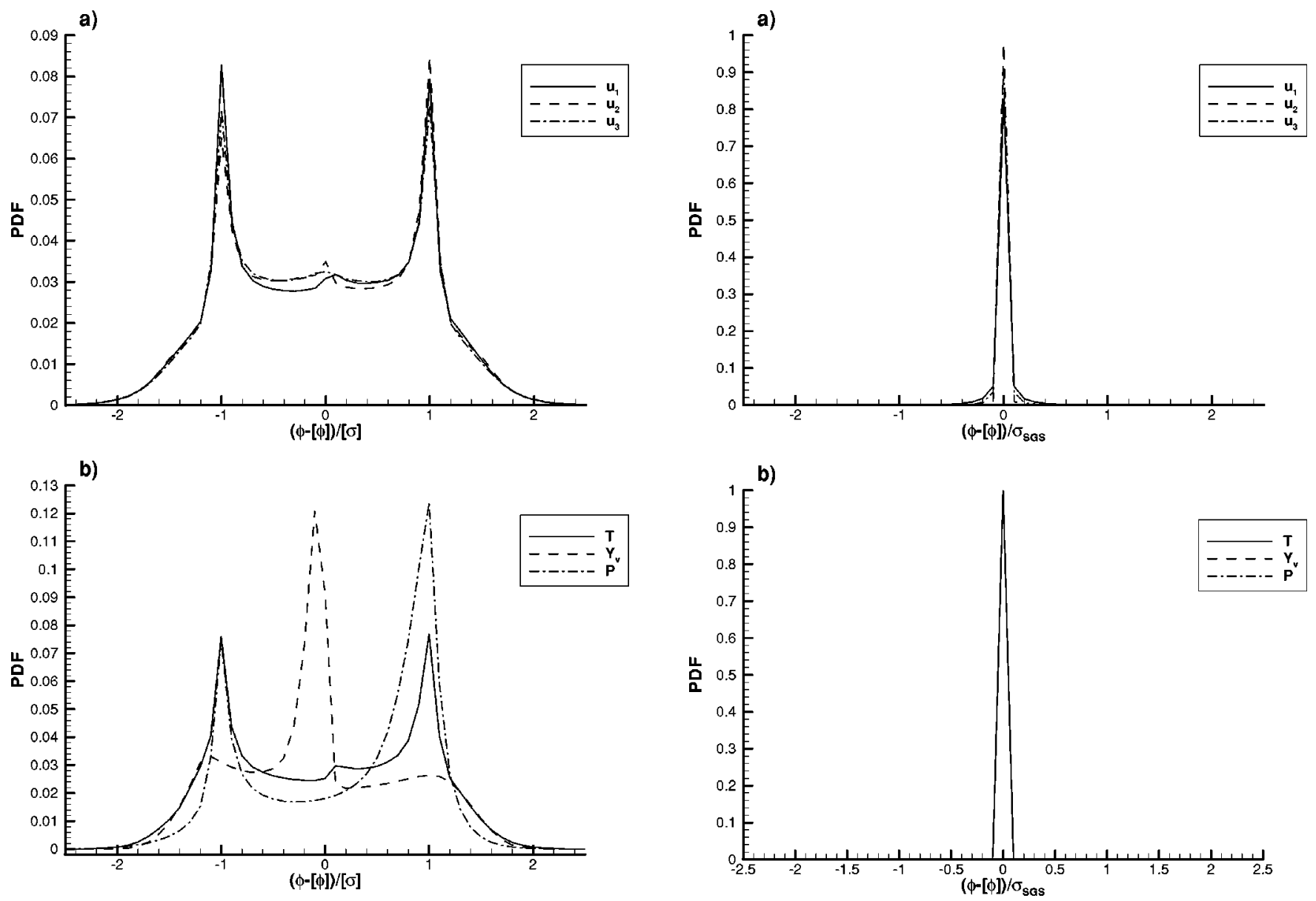

FIG. 7. PDF of $(\phi-\bar{\phi}) / \bar{\sigma}$ (a) $u_{1}, u_{2}, u_{3}$; (b) $T, P, Y_{V}$ ([ ] denotes filtering).

$$
\eta_{j}=C_{G} \Delta^{2} \frac{\partial \widetilde{Y}_{V}}{\partial x_{k}} \frac{\partial \widetilde{u}_{j}}{\partial x_{k}}
$$

is easily extended to compute the subgrid variances for any quantity $\phi$ as

$$
\sigma_{\mathrm{SGS}}^{2}(\phi)=\overline{\phi \phi}-\bar{\phi} \bar{\phi}=C_{G} \Delta^{2} \frac{\partial \bar{\phi}}{\partial x_{k}} \frac{\partial \bar{\phi}}{\partial x_{k}} .
$$

Theoretically, $C_{G} \Delta^{2}$ is the moment of the filtering volume $\left[I_{c} \Delta^{2}\right.$ of Eq. (53)] as can be seen by integrating the square of the Taylor expansion for $\phi$, Eq. (51), over the filtering volume, and using filtered instead of unfiltered quantities in the calculation of the derivatives. Thus, theoretically, for a cubic top-hat filter $C_{G}=\frac{1}{12}$.

This model is not only easily extended to calculate $\sigma_{\mathrm{SGS}}$ for all variables, but also has the advantage that the derivatives are already available from the computation of the resolved fields.

\section{Scale-similarity SGS model}

In the scale-similarity model, a second filter $\hat{\Delta}$ is introduced and the protocol involves refiltering the flow field ${ }^{34}$ with a test filter $\hat{\Delta} \geqslant \Delta$ (filtering at level $\hat{\Delta}$ is unweighted) to yield
FIG. 8. PDF of $(\phi-\bar{\phi}) / \sigma_{\mathrm{SGS}}$ (a) $u_{1}, u_{2}, u_{3}$; (b) $T, P, Y_{V}$ ([ ] denotes filtering).

$$
\begin{aligned}
& \tau_{i j}=C_{S}\left(\widehat{\widetilde{u}}_{i} \widehat{\tilde{u}}_{j}-\hat{\tilde{u}}_{i} \hat{\tilde{u}}_{j}\right), \\
& \theta_{j}=C_{S}\left(\widehat{\tilde{u}_{j} \bar{T}}-\hat{\tilde{u}}_{j} \hat{\bar{T}}\right), \\
& \eta_{j}=C_{S}\left(\widehat{\tilde{u}_{j} \tilde{Y}_{V}}-\hat{\tilde{u}}_{j} \hat{\tilde{Y}}_{V}\right) .
\end{aligned}
$$

A value of $C_{S}=1$ is necessary to assure Galilean invariance; if the model were completely exact, $C_{S}$ would also be 1 . Deviations from unity reflect therefore departures from the exact representation of $\tau_{i j}, \theta_{j}$, and $\eta_{j}$ computed from the DNS through Eqs. (68)-(70).

Similar to the gradient model, the scale-similarity model is easily extended to compute the subgrid variances for any quantity $\phi$ as

$$
\sigma_{\mathrm{SGS}}^{2}(\phi)=\overline{\phi \phi}-\bar{\phi} \bar{\phi}=C_{S}(\widehat{\bar{\phi} \bar{\phi}}-\hat{\bar{\phi}} \hat{\bar{\phi}}) .
$$

\section{Model coefficients}

In the following we will refer to standard deviations calculated from Eq. (55) as "exact" since in this model they represent the best available values that can be calculated at the LES (i.e., filtered) scale from the unfiltered variables. Therefore, we first compute the correlation between the "exact" and model SGS standard deviations using the definitions of Eqs. (45) and (46) and then determine the relation- 

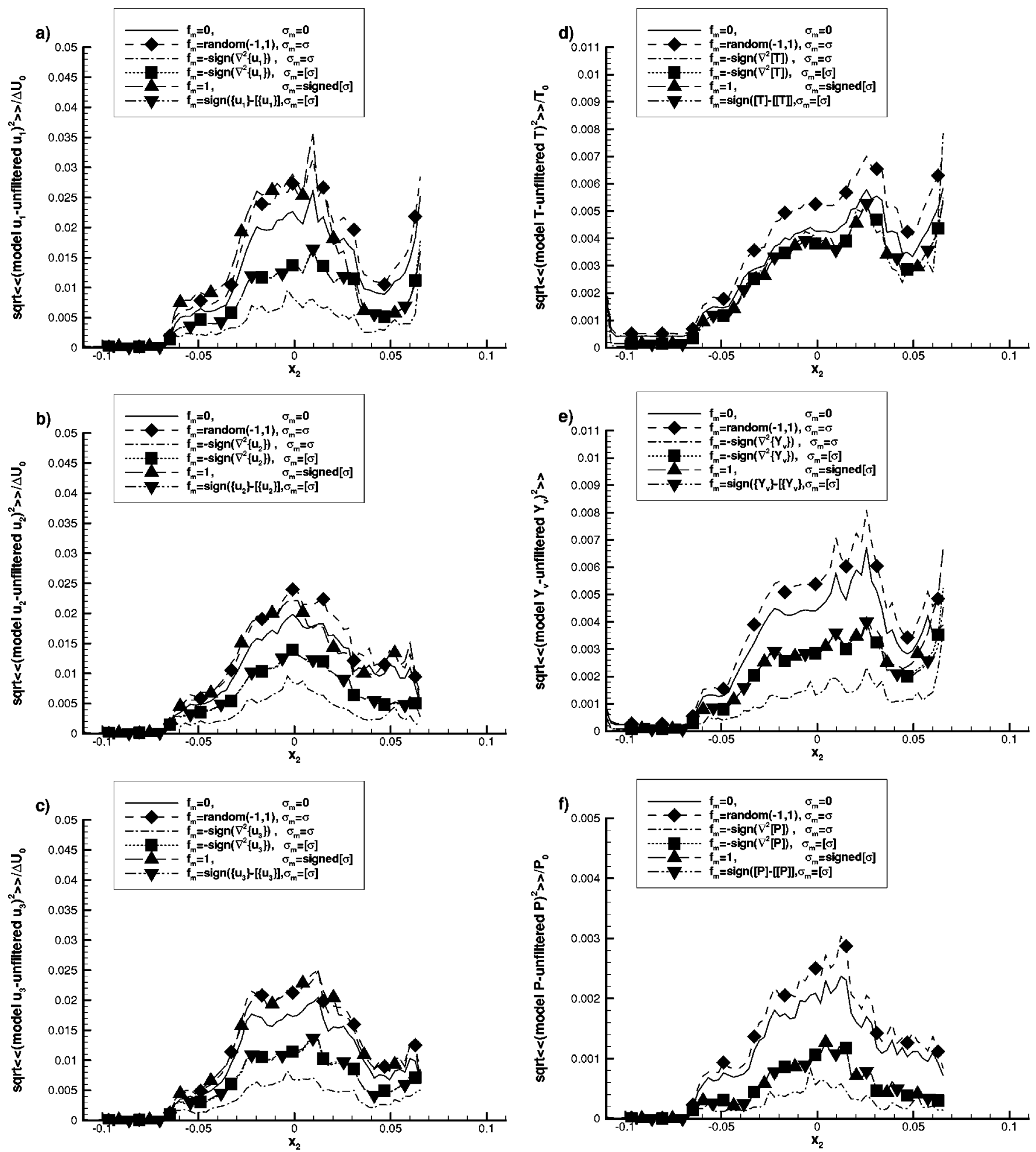

FIG. 9. Error in unfiltered variable model $\phi=\bar{\phi}+f_{m} \sigma_{m}$ interpolated to droplet locations, case TP600 (a) $u_{1}$; (b) $u_{2}$; (c) $u_{3}$; (d) $T$; (e) $Y_{V}$ (f) $P$ ([ ] and \{\} denote regular and Favre filtering, respectively).

ship between the "exact" and modeled variables which yields the model coefficient. Note that Eq. (45) allows pointwise assessment of the correlations.

The simplest SGS model is that having a constant coefficient with $C_{R}, C_{G}, C_{S}$ being the same for all flow variables over the entire (spatial and temporal) domain. For this type of model, the coefficient can be determined using a least-squares fit to $Y=b X$ which leads to $b$ $=\langle\langle\langle X Y\rangle\rangle\rangle /\langle\langle\langle X X\rangle\rangle\rangle$. If $X$ is the model standard deviation and $Y$ is the "exact" standard deviation, then $b$ is the square root of the model coefficient. More sophisticated models would have the model coefficients as functions of space and time; this will be the subject of a further investigation as we note that the simplicity of the constant coefficient models is 

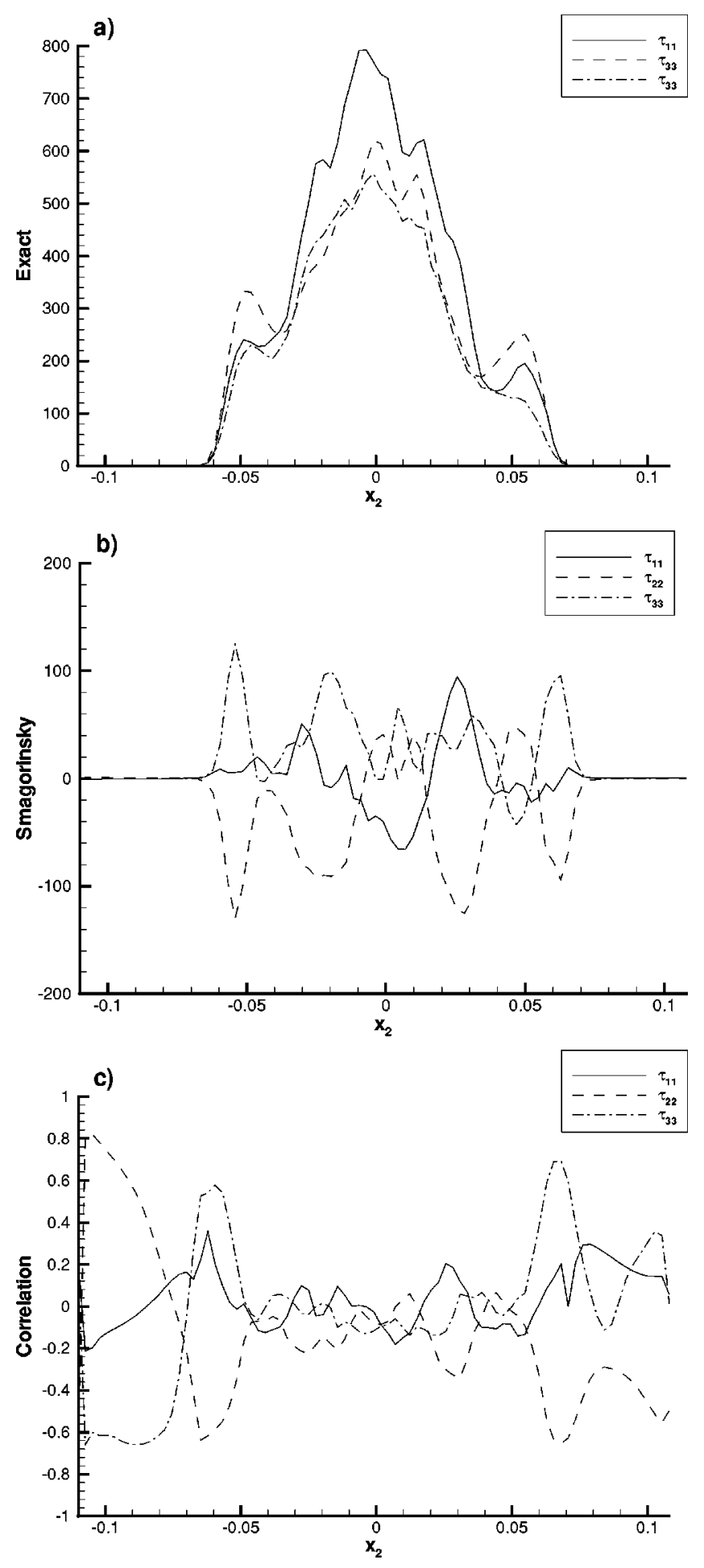

FIG. 10. Subgrid stresses, case TP600 (a) exact; (b) Smagorinsky; (c) correlation between exact and Smagorinksy stresses.

partially offset by the fact that they yield models which are flow dependent. However, considering the lack of experience with a priori models for two phase flows, it is worthwhile in this first step to assess the possibility of constant model coefficients since they are the simplest to derive and use in a LES.

\section{FINALIZATION OF THE QUANTITATIVE SGS MODELS}

\section{A. Unfiltered variable model}

As stated in Eq. (49), the unfiltered variable $\phi$ can be expressed as $\phi=\bar{\phi}+f \sigma$, where $\sigma=|\phi-\bar{\phi}|$ and $f=\operatorname{sign}(\phi$ $-\bar{\phi}$ ), and we showed in Eq. (59) a possible model for $f \sigma$ (denoted $f_{m} \sigma_{m}$ ) that has the potential of being more accurate than that obtained from a simple extrapolation of the filtered gas phase variables at the droplet locations (i.e., $f_{m} \sigma_{m}=0$ ). The proposed model will now be used to first compute $\phi$ for all dependent variables at the Eulerian grid points, and then interpolate it to the droplet locations.

Figure 7 shows the probability density function (PDF) for $(\phi-\bar{\phi}) / \bar{\sigma}$ for case TP600 with $\phi$ being $u_{1}, u_{2}, u_{3}, T$, $Y_{V}$, and $P$, where the sample space used in the calculation is the entire flow field (based on $\phi, \bar{\phi}$, and $\bar{\sigma}$ at each point), and where $\bar{\sigma}=\sqrt{\overline{\sigma^{2}}}$ is calculated from the filtered field using Eq. (58). Figure 8 shows the PDF for $(\phi-\bar{\phi}) / \sigma_{\mathrm{SGS}}$. Although the correlation between $\sigma$ and $\bar{\sigma}$ is similar to that between $\sigma$ and $\sigma_{\mathrm{SGS}}$ (ranging from 0.6 to 0.8 ), the ( $\phi$ $-\bar{\phi}) / \bar{\sigma}$ and $(\phi-\bar{\phi}) / \sigma_{\mathrm{SGS}}$ PDFs are remarkably different. The PDF for $(\phi-\bar{\phi}) / \sigma_{\mathrm{SGS}}$ shows one very large peak at zero, whereas that for $(\phi-\bar{\phi}) / \bar{\sigma}$ shows peaks at \pm 1 , similar to the PDF of $(\phi-\bar{\phi}) / \sigma$ which has values of \pm 1 . This confirms $\bar{\sigma}$ to be an appropriate model for $\sigma$, but implies that $\sigma$ and $\sigma_{\mathrm{SGS}}$ are only moderately correlated. Closer examination of the PDF of $(\phi-\bar{\phi}) / \bar{\sigma}$ in the $\left(x_{1}-x_{3}\right)$ plane (not shown) reveals the large $Y_{V}$ peak at zero to be due to the upper stream region where $Y_{V}=0$, whereas the small $u_{1}, u_{2}$, and $u_{3}$ peaks at zero correspond to the lower edge of the mixing layer, where free-stream droplets are being entrained. For all variables except $Y_{V}$, the PDFs of $(\phi-\bar{\phi}) / \bar{\sigma}$ in the dropletfree domain are focused on \pm 1 , but have a larger variation between -1 and +1 in the droplet-laden part.

Figure 9 shows the comparison between interpolating the gas dependent variables to the droplet locations using the exact quantities (unfiltered DNS), and interpolating the same variables using other models to the droplet locations. Results are presented in terms of the difference between the model and the unfiltered variable, averaged over droplets within a given $x_{2}$ interval and denoted by $\langle\langle\rangle\rangle$. In the first three models $\sigma_{m}=\sigma$ with $\sigma$ given by Eq. (48) (and therefore it is evaluated from the unfiltered variables which are not available in LES) with the purpose of comparing and evaluating the validity of the models for $f$. Clearly, $f_{m}=0$ at all locations leads to significant discrepancy between the exact and model interpolated variable. Additionally, any model with a mean $f_{m}=0$ will not perform any better since the deviations toward the unfiltered value will be just as likely as the deviations away from the unfiltered value. This is illustrated in Fig. 9 by the case where $f_{m}$ randomly assumes the values -1 or +1 ; its predictions generally lead to the largest deviations and its performance is slightly worse than $f_{m}=0$ despite having the exact $\sigma$. Using $f_{m}=-\operatorname{sign}\left(\nabla^{2} \bar{\phi}\right)$ gives significant improvement despite the two assumptions that $f_{m}=-\operatorname{sign}\left(\boldsymbol{\nabla}^{2} \phi\right)$ and $\operatorname{sign}\left(\boldsymbol{\nabla}^{2} \phi\right)=\operatorname{sign}\left(\boldsymbol{\nabla}^{2} \bar{\phi}\right)$; for the data 

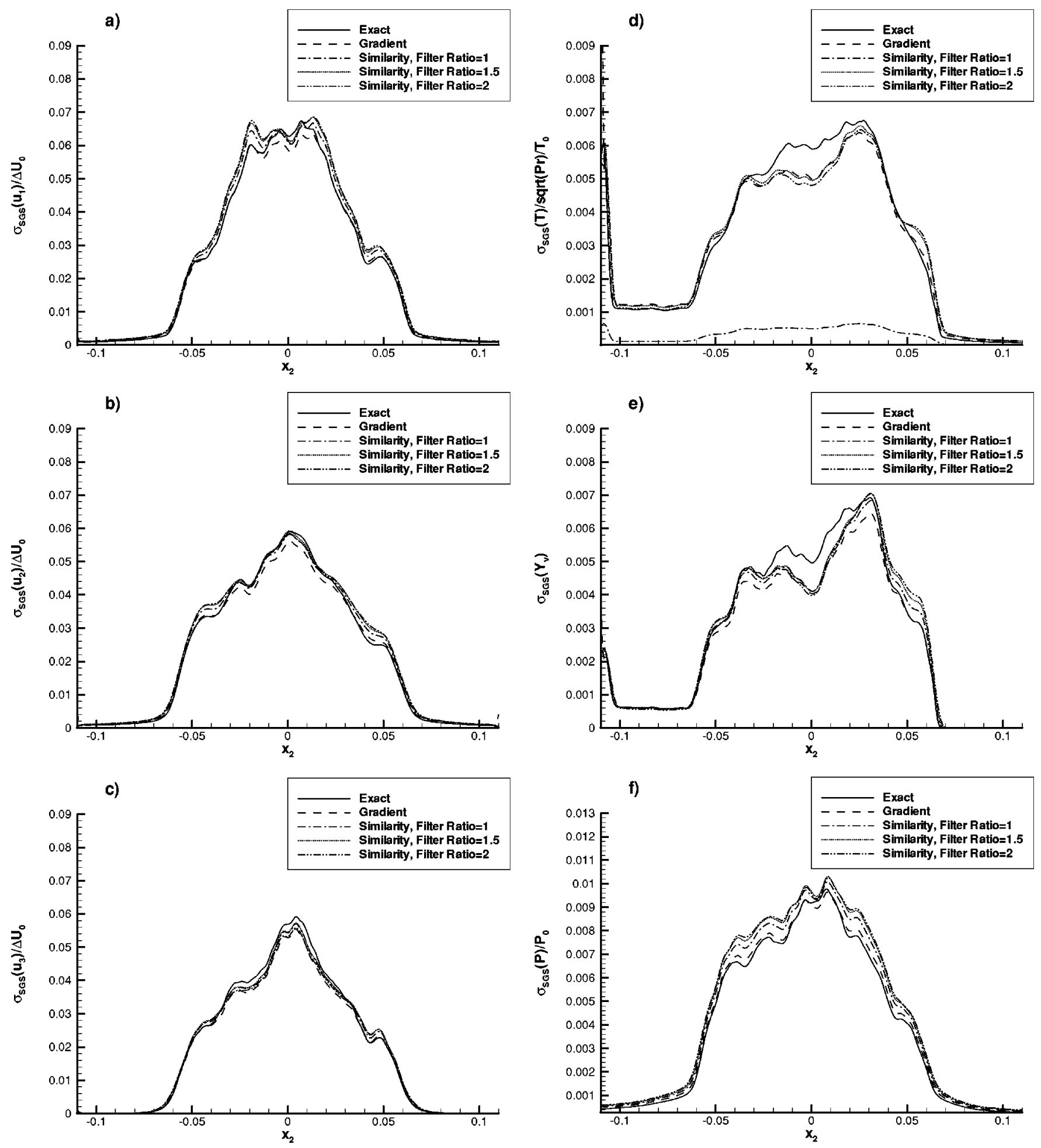

FIG. 11. SGS standard deviation, case TP500a (a) $u_{1}$; (b) $u_{2}$; (c) $u_{3}$; (d) $T$; (e) $Y_{V}$; (f) $P$.

shown in Fig. 9, these assumptions hold with $86 \%, 86 \%$, $86 \%, 85 \%, 86 \%$, and $93 \%$ certainty for $u_{1}, u_{2}, u_{3}, T, Y_{V}$, and $P$, respectively. When $\sigma$ is replaced by $\bar{\sigma}$ (denoted $[\sigma]$ in the figures), while $f_{m}=-\operatorname{sign}\left(\nabla^{2} \bar{\phi}\right)$, there is considerable improvement over the predictions obtained with $f_{m}$ $=0$, for all quantities except $T$ for which all models give similar results. For $T, Y_{V}$, and $P$, equivalent results are obtained with the two sets $\left(f_{m}=1, \sigma_{m}=\sqrt{\overline{\overline{\phi \phi}}}-\sqrt{\overline{\bar{\phi} \bar{\phi}}}\right)$ and $\left[f_{m}=-\operatorname{sign}\left(\nabla^{2} \bar{\phi}\right), \quad \sigma_{m}=\bar{\sigma}\right] \quad$ where $\quad \bar{\sigma}=\sqrt{\overline{\overline{\sigma^{2}}}}=\mid \sqrt{\overline{\phi \phi}}$ $-\sqrt{\overline{\bar{\phi}} \bar{\phi}} \mid$ is calculated as in Eq. (58); in Fig. 9 we have defined "signed $[\sigma]$ '" $=\sqrt{\overline{\bar{\phi} \phi}}-\sqrt{\overline{\bar{\phi} \bar{\phi}}}$. An alternative expression for $f_{m}, f_{m}=\operatorname{sign}(\phi-\bar{\phi})=\operatorname{sign}(\bar{\phi}-\overline{\bar{\phi}})$, is inspired by the scale-similarity relations and results in a similar accuracy to using $f_{m}=-\operatorname{sign}\left(\nabla^{2} \bar{\phi}\right)$ for all six variables.

The primary conclusion from the comparison of results 
a)

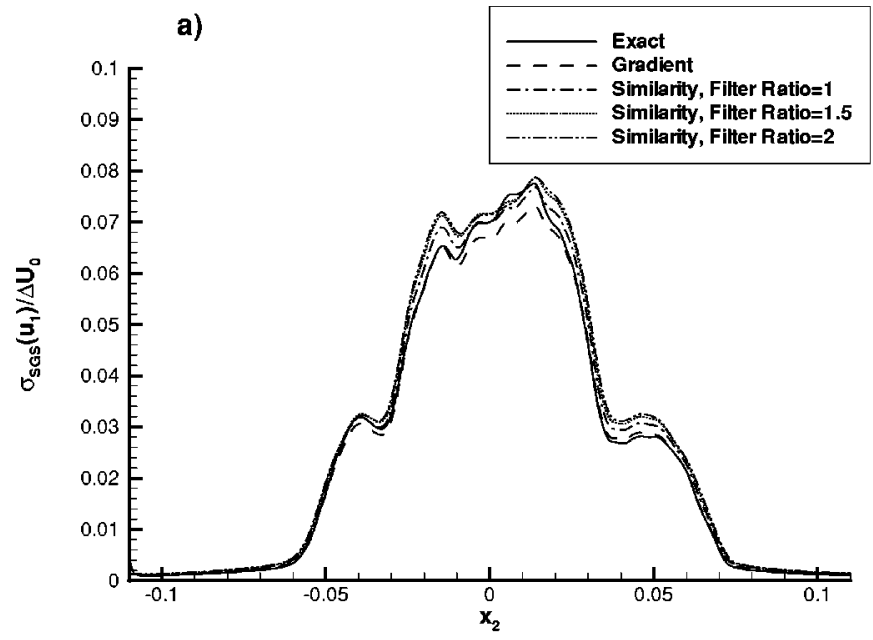

b)

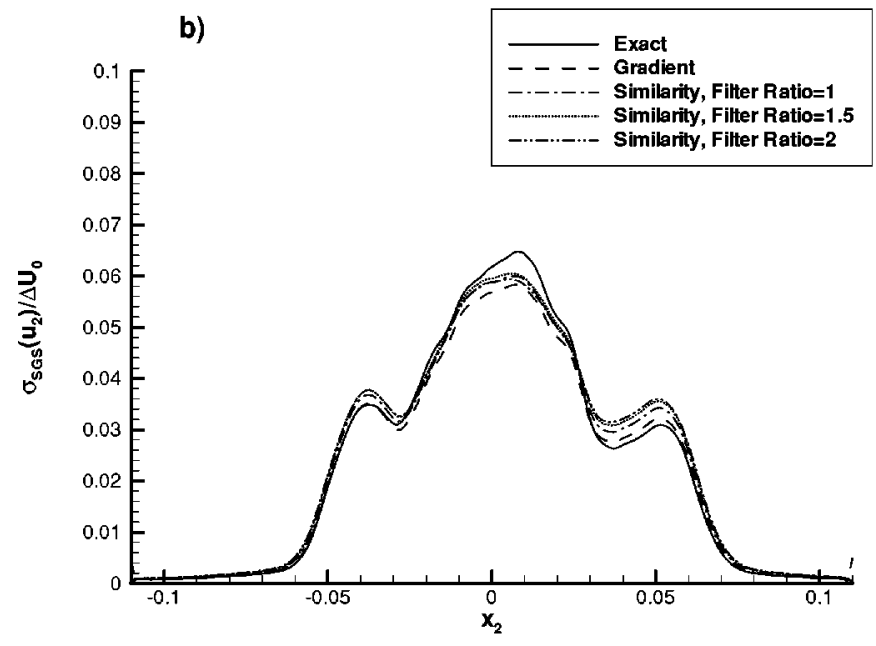

c)

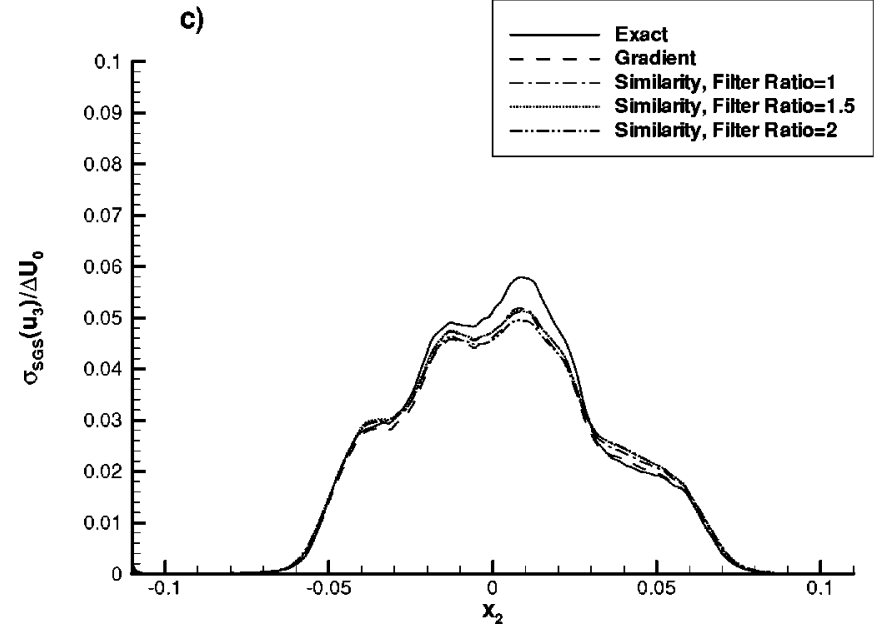

d)

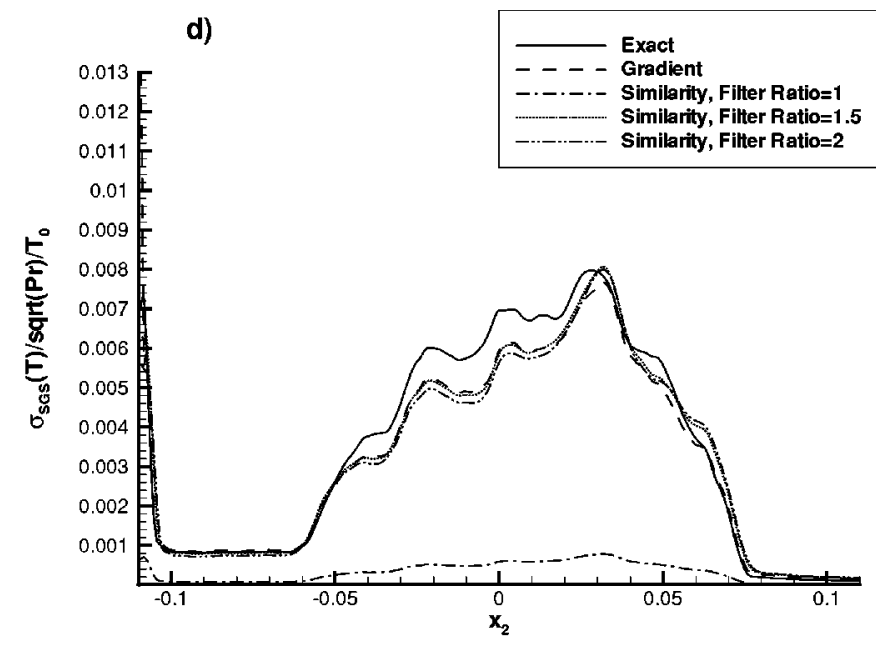

e)
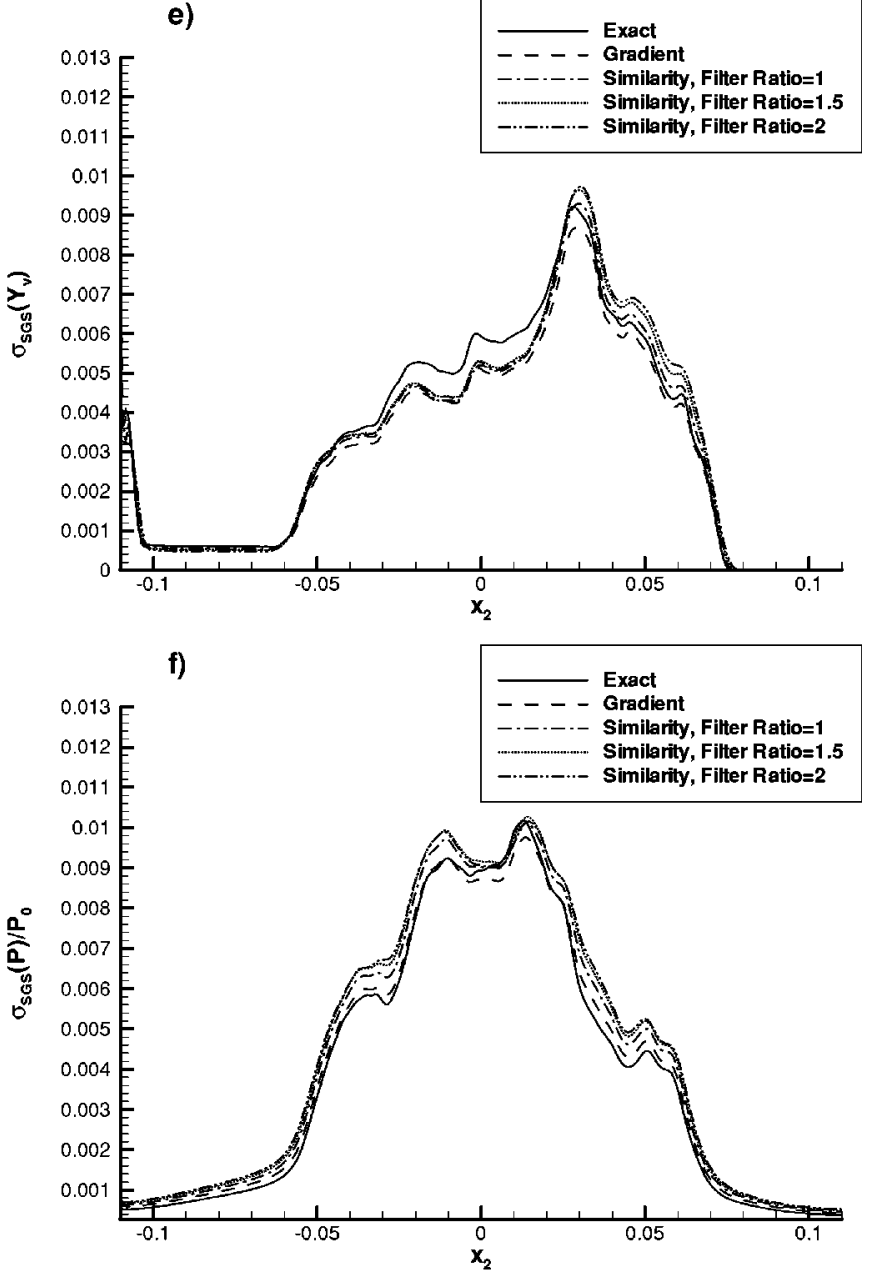

FIG. 12. SGS standard deviation, case TP500b (a) $u_{1}$; (b) $u_{2}$; (c) $u_{3}$; (d) $T$; (e) $Y_{V}$; (f) $P$.

obtained with modeled $f$ and $\sigma$ (the only possibility within LES) is that the "exact" model proposed in Eq. (59) is the most accurate at simulating the unfiltered gas phase variables as a function of the same filtered variables. Moreover, an analysis of the correlation $\mathcal{R}(\phi, \bar{\phi})$ defined by Eq. (57) shows that $\mathcal{R}=1$ for $T$ and $P, 0.97<\mathcal{R}<1$ for $u_{1}, u_{2}$, and $u_{3}$, with the greatest deviation from 1 appearing in the central part of the mixing layer; and $0.7<\mathcal{R}<1$ for $Y_{V}$, with the greatest deviation being obtained at the droplet-laden/ droplet-free interface. The low $Y_{V}$ correlation is due to the sparsity of drops, producing a $Y_{V}$ which reduces the quality of the statistics. Since apparently $\mathcal{R}<1$ for $Y_{V}$, we tried unsuccessfully to improve the modeling of the unfiltered quantities through the filtered quantities by slightly reducing $\mathcal{R}$. Not only was the model with $\mathcal{R}<1$ generally inferior, but even at the interface where the greatest deviation from unity 

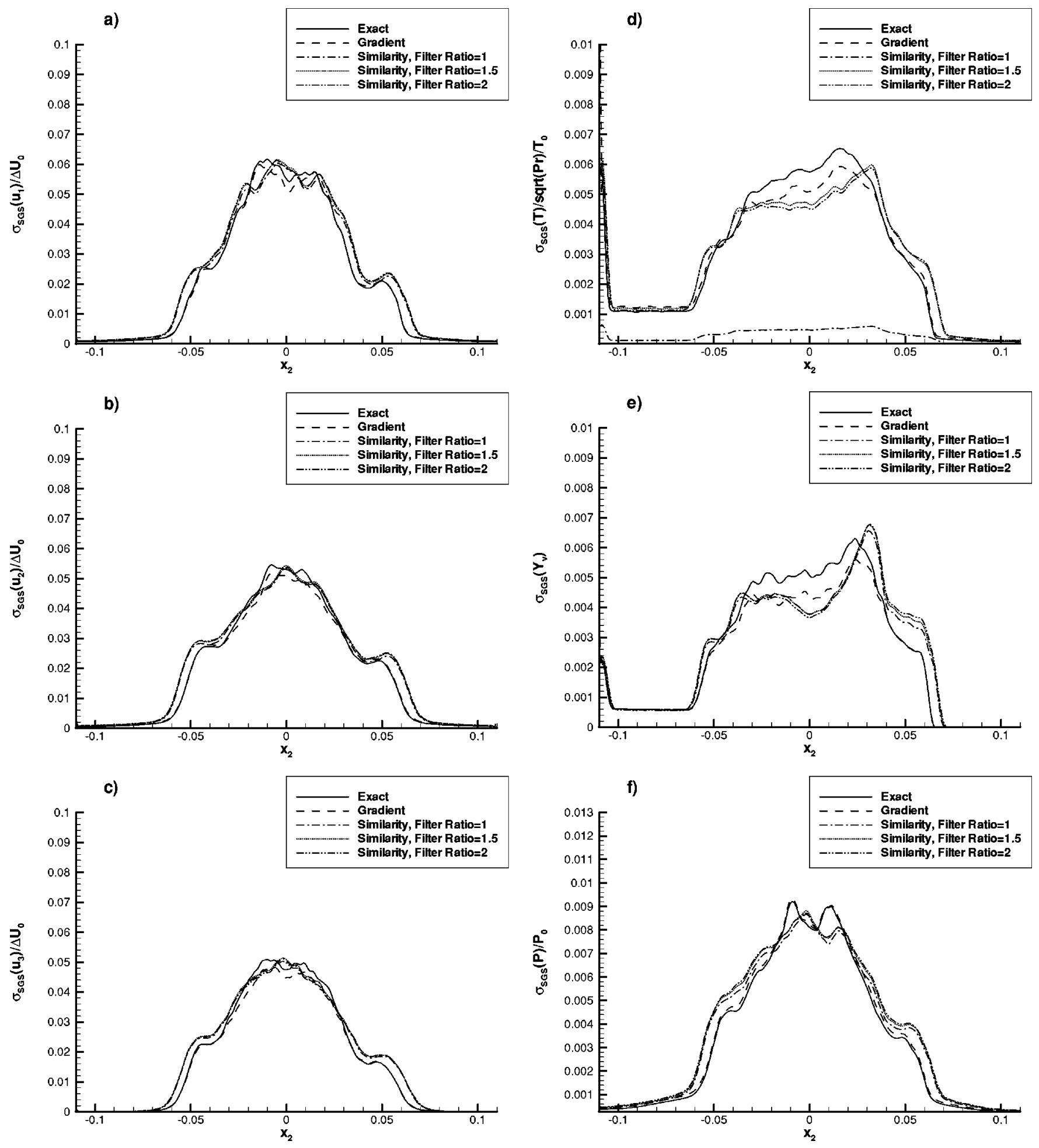

FIG. 13. SGS standard deviation, case TP600 (a) $u_{1}$; (b) $u_{2}$; (c) $u_{3}$; (d) $T$; (e) $Y_{V}$; (f) $P$.

was obtained, $\mathcal{R}=1$ led to the best prediction of $Y_{V}$ at droplet locations. Most important, $\mathcal{R}=1$ is the only value that provides the correct $\bar{\sigma} \simeq 0$ in the laminar free stream. Finally, we note that the efforts at reducing the error for the approximation of Eq. (59) through the reduction in the error in $Y_{V}$ are misplaced since the total model error is determined by that of the velocity components which are the largest (about $1.5 \%$ ); in contrast, the temperature, mass fraction, and pressure have errors an order of magnitude lower. Given these observations, it is unlikely that increased accuracy would be obtained even if a model with a variable $\mathcal{R}$ (according to the dependent variable) could be constructed. 
TABLE VII. Coefficient for gradient model.

\begin{tabular}{lllll}
\hline \hline & \multicolumn{1}{c}{ TP500a } & \multicolumn{1}{c}{ TP500b } & \multicolumn{1}{c}{ TP600 } & Mean \\
\hline$\Delta$ & $3.200 \times 10^{-3}$ & $3.200 \times 10^{-3}$ & $2.667 \times 10^{-3}$ & \\
$\sqrt{C_{G}}$ & 0.397 & 0.398 & 0.402 & 0.40 \\
\hline \hline
\end{tabular}

\section{B. Determination of SGS model constants}

Illustrated in Fig. 10 are the subgrid scale stresses calculated from the DNS, those predicted by the Smagorinsky model, and the correlation between the DNS calculated values and the model predictions calculated according to Eq. (45). The lack of good correlation between the DNS and predicted stresses is not surprising considering that even for single phase flows global correlations are in the range of 0.4 (see Refs. 35 and 22). However, in many single phase flows this model may still be acceptable because it provides the accurate global amount of dissipation. In two phase flows the modeling requirements are more stringent. Because source terms determining the interaction between phases must be locally calculated using the unfiltered variables, local rather than global correlations are of interest. Since the unfiltered variable model requires good local values of $\sigma_{\mathrm{SGS}}$ for computing $\bar{\sigma}$, the lack of good local correlation induced us to discard the Smagorinsky model.

Both the gradient and the scale-similarity models are assessed in Figs. 11-13 where the normalized $\sigma_{\mathrm{SGS}}$ planar averages are shown in Figs. 11, 12, and 13 for cases TP500a, TP500b, and TP600, respectively and $\sigma_{\mathrm{SGS}}$ is calculated either from the unfiltered values or using the models. For the sake of optimizing the scale-similarity model, we also consider several test-to-LES filter ratios, as the best value recommended in the literature is usually 2 (e.g., Ref. 22). The model coefficients used in all plots in Figs. 11-13 are the mean values $-C_{G}$ listed in Table VII for the gradient model and $C_{S}$ listed in Table VIII for the scale-similarity model. To find these model coefficients listed in Tables VII and VIII, linear fits of the exact $\sigma_{\mathrm{SGS}}$ to the model $\sigma_{\mathrm{SGS}}$ over the whole domain were performed. Since for the temperature the SGS deviations were underpredicted, the model coefficients were replaced by $C_{G} \mathrm{Pr}$ in Eq. (67) and $C_{S} \operatorname{Pr}$ in Eq. (71), where the Prandtl number is $\operatorname{Pr}=0.7$ for the present flow conditions. For all variables and all filter ratios of the scale-similarity model, as well as for the gradient model, the prediction of $\sigma_{\mathrm{SGS}}$ is very good, with that from the scale-similarity model being slightly superior. The only exception to this good agreement occurs for the temperature [see Figs. 11(d), 12(d), and $13(\mathrm{~d})]$ when the scale-similarity model with filter width ratio $\hat{\Delta} / \Delta=1$ is used. This is not completely surprising since

TABLE VIII. Coefficient for similarity model.

\begin{tabular}{ccccc}
\hline \hline$\hat{\Delta} / \Delta$ & TP500a & TP500b & TP600 & Mean $\sqrt{C_{S}}$ \\
\hline 1.0 & 1.237 & 1.253 & 1.243 & 1.24 \\
1.5 & 0.921 & 0.931 & 0.925 & 0.93 \\
2.0 & 0.751 & 0.759 & 0.755 & 0.75 \\
\hline \hline
\end{tabular}

as mentioned above, most investigators using a similarity model recommend $\hat{\Delta} / \Delta=2$.

Along with the individual SGS model constants for each set of DNS results, we also show in Tables VII and VIII the recommended mean constants for all runs. For the gradient model, the mean value of $\sqrt{C_{G}}=0.4$ is approximately $40 \%$ larger than the theoretical value of $\sqrt{C_{G}}=1 / \sqrt{12} \simeq 0.29$ for the cubic filter. The discrepancy between the model and theoretical values is attributed to departures from the behavior assumed by the gradient model, most notably to the replacement of unfiltered by filtered quantities in the calculation of the derivatives (as stated when formulating the model). For the scale-similarity model, a linear best fit gives $\sqrt{C_{S}}=1.71$ $-0.49(\hat{\Delta} / \Delta)$, however, with only three values for this fit, it is recommended to use the computed coefficients rather than the linear best fit. In particular, for $\hat{\Delta} / \Delta=1$ we find that $\sqrt{C_{S}} \neq 1$, indicating that $\overline{\bar{\phi}}=\bar{\phi}$ is not a good assumption. Consistent with the previous discussion on the model for the unfiltered variables using the filtered variables, Figs. 11-13 show that the $\sigma_{\mathrm{SGS}}$ are largest for the velocity components whereas those for $Y_{V}, T$, and $P$ are an order of magnitude smaller.

\section{CONCLUSIONS}

An a priori subgrid analysis has been presented for a temporally developing mixing layer having one stream initially laden with a dilute collection of evaporating droplets. To construct the SGS model we employed a transitional DNS database for three initial Reynolds numbers (based on vorticity thickness) and mass loading combinations: (500; $0.2),(500 ; 0.5)$, and $(600 ; 0.2)$. The DNS equations were filtered, and LES equations were constructed subject to several assumptions. These assumptions were evaluated by calculating the exact filtered expressions and their filtered models for the three sets of results in the database, as well as the other terms in the equations. The comparison among these terms showed that the assumptions used to derive the final set of LES equations were well justified. For example, an order of magnitude analysis of the terms in these equations showed that the subgrid terms arising from filtering the inviscid terms are smaller only than the inviscid terms based on filtered quantities. However, the subgrid terms arising from filtering the viscous terms were found to be negligible. The triple correlation of velocity, mass fraction and temperature is found to be well modeled as the temperature multiplied by the mass-fraction-velocity correlation.

Within the LES formalism for two phase flows, it was found that there are two modeling issues in addition to the usual modeling of the SGS terms. One of these modeling issues, which is that the unfiltered gas phase dependent variables at the drop locations must be derived from the LES filtered variables, was addressed as a preliminary to SGS modeling; the knowledge of these unfiltered values is necessary for the accurate calculation of the source terms. For each gas phase variable, several constructs for the unfiltered value as function of the filtered value were considered, and it was shown that the most accurate is that with a standard 
deviation having the magnitude of the filtered standard deviation and the opposite sign of the Laplacian of the filtered variable. With this model, predictions for the unfiltered variables at the droplet locations were found to be considerably improved compared to simply interpolating the filtered variables.

To model the subgrid scale (SGS) standard deviations, two models, the gradient and scale-similarity, were found to give excellent results when the model constant was properly calculated using the database, while the traditional Smagorinsky model was found inadequate. More sophisticated dynamic models, where the present constant is replaced by a functional dependence of the filtered variables will be considered in the future. Statistical analysis of the database showed that the gradient model is well represented by the same model constant for all three cases. For the similarity model, the constant was found, as expected, to be dependent on the test-to-LES filter ratio.

Future work will address dynamic SGS modeling as well as the second issue in two phase flow LES calculations which is that of the computation of the averaged source terms from the filtered dependent variables. Once this issue is resolved, we will conduct an a posteriori testing of the gradient and scale-similarity models in an LES.

\section{ACKNOWLEDGMENTS}

This research was conducted at the Jet Propulsion Laboratory (JPL) of the California Institute of Technology (Caltech) under the sponsorship of General Electric (GE) through the Air Force Office of Scientific Research (AFOSR) Focused Research Initiative program with Dr. Hukam Mongia from GE serving as contract monitor. Computations were performed using the Caltech Center for Advanced Computing Research (CACR) parallel HP Exemplar.

${ }^{1}$ C. T. Crowe, T. R. Troutt, and J. N. Chung, "Numerical models for two-phase turbulent flows," Annu. Rev. Fluid Mech. 28, 11 (1996).

${ }^{2}$ M. Boivin, O. Simonin, and K. D. Squires, "Direct numerical simulation of turbulence modulation by particles in isotropic turbulence," J. Fluid Mech. 375, 235 (1998).

${ }^{3}$ S. Elghobashi and G. C. Truesdell, "On the two-way interaction between homogeneous turbulence and dispersed solid particles. I: Turbulence modification," Phys. Fluids A 5, 1790 (1993).

${ }^{4}$ K. D. Squires and J. K. Eaton, "Particle response and turbulence modification in isotropic turbulence," Phys. Fluids A 2, 1191 (1990).

${ }^{5}$ A. Ansari and W. Z. Strang, Large-eddy simulation of turbulent mixing layers, Technical Report No. 96-0684, AIAA, 1996.

${ }^{6}$ B. Vreman, B. Geurts, and H. Kuerten, "Large-eddy simulation of the turbulent mixing layer," J. Fluid Mech. 339, 357 (1997).

${ }^{7}$ E. Deutsch and O. Simonin, "Large eddy simulation applied to the modelling of particulate transport coefficients in turbulent two-phase flows," Eighth Symposium on Turbulent Shear Flows 10-1, 1 (1991).

${ }^{8}$ O. Simonin, E. Deutsch, and M. Boivin, "Large eddy simulation and second-moment closure of particle fluctuating motion in two-phase turbulent shear flows," Turbulent Shear Flows 9, 85 (1993).

${ }^{9}$ Q. Wang, K. D. Squires, and O. Simonin, "Large eddy simulation of turbulent gas-solid flows in a vertical channel and evaluation of secondorder models," Int. J. Heat Fluid Flow 19, 505 (1998).
${ }^{10} \mathrm{~F}$. Yeh and U. Lei, "On the motion of small particles in a homogeneous turbulent shear flow," Phys. Fluids A 3, 2758 (1991).

${ }^{11} \mathrm{Q}$. Wang and K. D. Squires, "Transport of heavy particles in a threedimensional mixing layer," J. Fluids Eng. 120, 613 (1998).

${ }^{12}$ W. S. J. Uijttewaal and R. V. A. Oliemans, "Particle dispersion and deposition in direct numerical and large eddy simulations of vertical pipe flows," Phys. Fluids 8, 2590 (1996).

${ }^{13} \mathrm{Q}$. Wang and K. D. Squires, "Large eddy simulation of particle deposition in a vertical turbulent channel flow," Int. J. Multiphase Flow 22, 667 (1996).

${ }^{14} \mathrm{Q}$. Wang and K. D. Squires, "Large eddy simulation of particle-laden turbulent channel flow," Phys. Fluids 8, 1207 (1996).

${ }^{15} \mathrm{~S}$. Pannala and S. Menon, "Large eddy simulations of two-phase turbulent flows," Technical Report No. AIAA 98-0163, 1998.

${ }^{16}$ F. F. Grinstein and K. Kailasanath, "Three-dimensional numerical simulations of unsteady reactive square jets," Combust. Flame 100, 2 (1995).

${ }^{17}$ D. Hansell, I. M. Kennedy, and W. Kollmann, "A simulation of particle dispersion in a turbulent jet," Int. J. Multiphase Flow 18, 559 (1992).

${ }^{18} \mathrm{~F}$. Mashayek, "Droplet-turbulence interactions in low-Mach-number homogeneous shear two-phase flows," J. Fluid Mech. 367, 163 (1998).

${ }^{19}$ R. S. Miller and J. Bellan, "Direct numerical simulation and subgrid analysis of a transitional droplet laden mixing layer,' Phys. Fluids 12, 650 (2000).

${ }^{20}$ R. S. Miller and J. Bellan, "Direct numerical simulation of a confined three-dimensional gas mixing layer with one evaporating hydrocarbondroplet laden stream," J. Fluid Mech. 384, 293 (1999).

${ }^{21}$ G. C. Truesdell and S. Elghobashi, "On the two-way interaction between homogeneous turbulence and dispersed solid particles. II. Particle dispersion," Phys. Fluids A 6, 1405 (1994).

${ }^{22}$ B. Vreman, B. Geurts, and H. Kuerten, "A priori tests of large eddy simulation of the compressible plane mixing layer,' J. Eng. Math. 29, 299 (1995).

${ }^{23}$ R. S. Miller, K. Harstad, and J. Bellan, "Evaluation of equilibrium and non-equilibrium evaporation models for many-droplet gas-liquid flow simulations,"' Int. J. Multiphase Flow 24, 1025 (1998).

${ }^{24} \mathrm{X}$-L. Tong and L-P. Wang, "Direct simulations of particle transport in two and three dimensional mixing layers," ASME Fluids Engineering Division Summer Meeting, paper 3635, June 22-26, 1997.

${ }^{25}$ R. D. Moser and M. M. Rogers, "Mixing transition and the cascade to small scales in a plane mixing layer," Phys. Fluids A 3, 1128 (1991).

${ }^{26}$ T. J. Poinsot and S. K. Lele, "Boundary conditions for direct simulations of compressible viscous flows," J. Comput. Phys. 101, 104 (1992).

${ }^{27}$ S. Ghosal, "Mathematical and physical constraints on Large-eddy simulation of turbulence," AIAA J. 37, 425 (1999).

${ }^{28}$ G. Erlebacher, M. Y. Hussaini, C. G. Speziale, and T. A. Zang, "Toward the large-eddy simulation of compressible turbulent flows," J. Fluid Mech. 238, 155 (1992).

${ }^{29}$ J. Domaradzki and K. Loh, "The subgrid-scale estimation model in the physical space representation," Phys. Fluids 11, 2330 (1999).

${ }^{30}$ J. Kuerten, B. Geurts, A. Vreman, and M. Germano, "Dynamic inverse modeling and its testing in large-eddy simulations of the mixing layer," Phys. Fluids 11, 3778 (1999).

${ }^{31}$ K.-C. Loh and J. Domaradzki, "The subgrid-scale estimation model on nonuniform grids,' Phys. Fluids 11, 3786 (1999).

${ }^{32}$ J. Smagorinksy, Some historical remarks on the use of nonlinear viscosities, in Large Eddy simulation of complex engineering and geophysical flows, edited by B. Galperin and S. A. Orszag (Cambridge University Press, Cambridge, UK, 1993); Chaps. 1 and 3.

${ }^{33}$ S. Liu, C. Meneveau, and J. Katz, "On the properties of similarity subgrid-scale models as deduced from measurements in a turbulent jet," $\mathrm{J}$. Fluid Mech. 275, 83 (1994)

${ }^{34}$ J. Bardina, J. H. Ferziger, and W. C. Reynolds, "Improved subgrid scale models for large eddy simulation," Technical Report No. AIAA 80-1357 (1980).

${ }^{35}$ R. A. Clark, J. H. Ferziger, and W. C. Reynolds, "Evaluation of subgridscale models using an accurately simulated turbulent flow," J. Fluid Mech. 91, 1 (1979). 